Probing Higgs-radion mixing in warped models through complementary searches at the LHC and the ILC

\title{
Frank, Mariana
}

2016-09-12

Frank , M , Huitu , K, Maitra , U \& Patra , M 2016 , ' Probing Higgs-radion mixing in warped models through complementary searches at the LHC and the ILC ' , Physical Review D, vol. 94 , no. 5 , 055016 . https://doi.org/10.1103/PhysRevD.94.055016

http://hdl.handle.net/10138/183651

https://doi.org/10.1103/PhysRevD.94.055016

cc_by

publishedVersion

Downloaded from Helda, University of Helsinki institutional repository.

This is an electronic reprint of the original article.

This reprint may differ from the original in pagination and typographic detail.

Please cite the original version. 


\title{
Probing Higgs-radion mixing in warped models through complementary searches at the LHC and the ILC
}

\author{
Mariana Frank, ${ }^{1, *}$ Katri Huitu, ${ }^{2, \dagger}$ Ushoshi Maitra, ${ }^{3, \$}$ and Monalisa Patra ${ }^{4,8}$ \\ ${ }^{1}$ Department of Physics, Concordia University, 7141 Sherbrooke St. West, Montreal, Quebec, \\ Canada H4B $1 R 6$ \\ ${ }^{2}$ Department of Physics and Helsinki Institute of Physics, P.O. Box 64 (Gustaf Hällströminkatu 2), \\ University of Helsinki, Helsinki FIN-00014, Finland \\ ${ }^{3}$ Department of Theoretical Physics, Tata Institute of Fundamental Research, Mumbai 400 005, India \\ ${ }^{4}$ Ruđjer Bošković Institute, Division of Theoretical Physics, Bijenička 54, HR-10000 Zagreb, Croatia
}

(Received 4 July 2016; published 12 September 2016)

\begin{abstract}
We consider the Higgs-radion mixing in the context of warped space extradimensional models with custodial symmetry and investigate the prospects of detecting the mixed radion. Custodial symmetries allow the Kaluza-Klein excitations to be lighter and protect $Z b \bar{b}$ to be in agreement with experimental constraints. We perform a complementary study of discovery reaches of the Higgs-radion mixed state at the 13 and $14 \mathrm{TeV}$ LHC and at the 500 and $1000 \mathrm{GeV}$ International Linear Collider (ILC). We carry out a comprehensive analysis of the most significant production and decay modes of the mixed radion in the $80 \mathrm{GeV}-1 \mathrm{TeV}$ mass range and indicate the parameter space that can be probed at the LHC and the ILC. There exists a region of the parameter space which can be probed, at the LHC, through the diphoton channel even for a relatively low luminosity of $50 \mathrm{fb}^{-1}$. The reach of the four-lepton final state in probing the parameter space is also studied in the context of $14 \mathrm{TeV} \mathrm{LHC}$, for a luminosity of $1000 \mathrm{fb}^{-1}$. At the ILC, with an integrated luminosity of $500 \mathrm{fb}^{-1}$, we analyze the $Z$-radion associated production and the $W W$ fusion production, followed by the radion decay into $b \bar{b}$ and $W^{+} W^{-}$. The $W W$ fusion production is favored over the $Z$-radion associated channel in probing regions of the parameter space beyond the LHC reach. The complementary study at the LHC and the ILC is useful both for the discovery of the radion and the understanding of its mixing sector.
\end{abstract}

DOI: $10.1103 /$ PhysRevD.94.055016

\section{INTRODUCTION}

The Standard Model (SM) of particle physics has been successful in describing the fundamental particles of our world and is currently in good agreement with almost all the experimental results. However, under closer scrutiny, the SM reveals that there are well-motivated reasons for expecting new physics. The most discussed topic in the area of theoretical shortcomings of the SM is the so-called hierarchy problem. In a nutshell, the problem is that the SM fails to explain why the Higgs mass is light (electroweak scale), while calculations within the SM framework allow it to grow indefinitely to Planck scales. Many theoretical frameworks were proposed to take into account and resolve the hierarchy problem. Supersymmetry and extradimensional models are the most studied scenarios in the literature. In this analysis, we will work in the context of the Randall-Sundrum (RS) warped extradimensional model.

The solution to the gauge hierarchy problem in the context of extra dimensions, earlier suggested by Arkani-

\footnotetext{
*mariana.frank@concordia.ca

†katri.huitu@helsinki.fi

*ushoshi@theory.tifr.res.in

§mpatra@irb.hr
}

Hamed, Dimopoulos, and Dvali (ADD) [1-3] allowed only gravity to access the extra dimension(s). The ADD scenario explained the weakness of the gravity compared to other forces by the fact that gravity becomes diluted in the volume of large extra dimensions. However, this scenario transferred the gauge hierarchy problem onto the problem of the discrepancy between the large size of extra dimensions, $\mathrm{R} \approx 1 \mathrm{~mm}$ (fixed from experimental constraints), and the natural value of $R \approx 10^{-33} \mathrm{~cm}$. An ambitious proposal to the hierarchy problem was then put forward by Randall and Sundrum. In the original RS model [4], there are two 3-branes embedded in the five-dimensional (5D) anti-de Sitter (AdS) space, with all the SM particles localized on the visible 3-brane and only the graviton propagating in the bulk. This model offers a simple and natural solution to the hierarchy problem. The separation between the two 3-branes leads directly to the existence of an additional scalar called the radion, corresponding to the quantum fluctuations of the distance between the two 3-branes. The radion is massless in the limit of the backreaction going to zero but acquires a mass with a suitable stabilizing mechanism [5]. The radion can be much lighter than the massive gravitons. For different phenomenological studies, the mass of the radion $m_{\varphi}$ is usually considered in the range of $\mathcal{O}(10 \mathrm{GeV}) \leq m_{\varphi} \leq \mathcal{O}(\mathrm{TeV})$ [6]. The radion couples 
with the matter via the trace of the energy momentum tensor. Thus, the structure of the coupling of the radion with the SM fields is similar to that of the Higgs boson. However, for massless gauge bosons, there is an enhancement coming from the trace anomaly term. General covariance allows a possibility of mixing between the radion and the Higgs boson. The dedicated analysis of the Higgs sector at the LHC will help in constraining the Higgs-radion mixing hypotheses. The phenomenology of the Higgs-radion mixed sector has been studied thoroughly in the literature in the context of the electroweak precision measurements and using the Higgs results from the LHC [7-20].

In an effort to improve the predictions of the model, and to render it suitable for phenomenology, the RS model was modified, allowing the gauge bosons to propagate in the bulk, so as to facilitate the gauge coupling unification [21]. Allowing the gauge bosons in the bulk in turn introduces towers of gauge Kaluza-Klein (KK) states on the TeV brane. The SM fermions and the Higgs, localized on the $\mathrm{TeV}$ brane couple maximally to these KK gauge states, leading to large corrections to the Peskin-Takeuchi ( $S$ and $T)$ parameters, respectively. This in turn leads to strong constraints on the $5 \mathrm{D}$ theory from the electroweak precision observables. The bounds on the KK masses are of the order of $30 \mathrm{TeV}$ [22-26] in the models with only the SM gauge bosons and the gravitons in the bulk. Putting the SM fermions in the bulk along with the massive SM gauge bosons solves the flavor violation problems of the theory [27]. The Higgs boson is localized on the IR brane in this modified model, to account for the gauge hierarchy problem. This approach naturally explains the hierarchy problem in the Yukawa sector, with the fermions being localized at different points in the bulk. Localizing lighter fermions nearer to the Planck brane, with the top and the Higgs localized on the IR brane, leads to weakening of constraints on the KK gauge states from the oblique $S$ parameter. However, the strong constraints from the $T$ parameter still persist [28], with the electroweak precision results imposing a bound of around $10 \mathrm{TeV}$ on the mass of lightest spin-1 resonances. This strong constraint from $T$ parameter in these 5D warped bulk scenarios can be mitigated by various methods, discussed in Ref. [29]. One of the possible cures for the increased $T$ parameter is to extend the symmetry of the model to the custodial $S U(2)_{R}$ symmetry, first discussed in Ref. [30]. The bulk gauge symmetry in this case is $S U(3)_{c} \times S U(2)_{L} \times$ $S U(2)_{R} \times U(1)_{X}$. The tree-level $S$ and $T$ parameters in this scenario are well behaved, with the $T$ parameter likely to vanish and the constraints mainly coming from the $S$ parameter. This in turn leads into a lower bound of about $6 \mathrm{TeV}$ on the first $\mathrm{KK}$ mode of the gauge bosons. The second possible solution which limits the masses of the first $\mathrm{KK}$ gauge bosons to the order of $5 \mathrm{TeV}$ is the one with large brane localized kinetic terms for the gauge fields $[31,32]$.
The third possibility is in the form of the models with deformed metric [33-36]. In this framework, the SM particles, including the Higgs, are in the bulk, and the space departs from $\mathrm{AdS}_{5}$ near the IR brane, while being similar to RS near the UV brane. In these scenarios, KK gauge boson modes as low as $1-2 \mathrm{TeV}$ can be consistent with the electroweak precision tests, for a suitable choice of model parameters. Recent works discussing the bounds on the lightest KK gauge boson, in the context of the above scenarios, with up to date electroweak fits are in Ref. [37].

Here, we choose to work in the framework of custodial RS model and concentrate on the phenomenological aspect of the Higgs-radion mixing, where all the SM particles except the Higgs bosons are in the bulk. The fermions are allowed to be localized anywhere in the bulk, leading to the natural generation of the Yukawa coupling hierarchies. The fermion masses depend on the bulk mass parameters $c_{L}$ (for the doublets) and $c_{R}$ (for right-handed fields) characterizing the profiles of the zero mode fermions. The values of $c_{L}$ and $c_{R}$ are fixed depending on the wave function of the fermion on the Planck brane or the $\mathrm{TeV}$ brane. They are chosen to match the fermion mass hierarchy fixed through the physical quark masses and mixing at the weak scale. The custodial RS model, compared to the original RS model, predicts the existence of five additional gauge bosons, three of which are neutral and two of which are charged. The coupling of the radion with the SM gauge bosons (massive and massless) in the custodial RS model is similar to the noncustodial case. The top quark being heavy (localized on the $\mathrm{TeV}$ brane like the new $\mathrm{KK}$ modes) couples strongly to the new heavy gauge bosons. The bottom quark $\left(b_{L}\right)$ being in the same electroweak doublet as $t_{L}$ is also affected and in turn modifies the $Z b_{L} \bar{b}_{L}$ coupling, which must be in accordance with the SM prediction at the $0.25 \%$ level. This calls for adjusting the profiles for $(t, b)_{L}$ and $t_{R}$, so as to protect the $Z b \bar{b}$ coupling. There are many analyses which have looked into different fermion representations, with the custodial symmetry protecting $Z b \bar{b}$ [38-40]. These scenarios consider the first two generations of the quarks and the leptons as doublets under the $S U(2)_{L}$. The third generation left-handed quarks are in the doublet representation of both $S U(2)_{L}$ and $S U(2)_{R}$, which leads to non-SM fermions with no zero modes. These fermions are very heavy and therefore not relevant for our analysis. In order to accommodate the large top and bottom mass difference, $t_{R}$ can be either in a singlet or in a triplet representation of $S U(2)_{R}$ (for details, see Refs. [38,40]). Overall, the top and the bottom quark can have various possible quantum numbers and profiles in the extra dimension, so as to satisfy the constraints from flavor violation and precision tests. Different possibilities include the localization of $t_{R}$ very close to the $\mathrm{TeV}$ brane with $(t, b)_{L}$ having a profile close to flat or the contrary case with $(t, b)_{L}$ very close to the TeV brane and $t_{R}$ close to flat. The intermediate case with the profile of $(t, b)_{L}$ and $t_{R}$ 
being near, not too close to, the $\mathrm{TeV}$ brane is also possible. It was shown in Refs. $[39,41]$ that the electroweak fits favor a close to flat profile for $(t, b)_{L}$, with $t_{R}$ peaked near the $\mathrm{TeV}$ brane. Therefore, we consider this case, with $c_{L}^{(t, b)}=$ $0.4-0.3$ and $c_{R}^{t}=0$. All the other fermions including $b_{R}$ are assumed to be localized on or very near the Planck brane, with $c_{L}^{i}>1 / 2, c_{R}^{i}<-1 / 2$. The specific choice of representations of the third generation quarks will not affect our results for $g g, \gamma \gamma, W^{+} W^{-}$final states, while the values for $c_{L}^{(t, b)}$ and $c_{R}^{(b)}$ would influence the analysis in the $b \bar{b}$ final state, but not by more than $\mathcal{O}(1)$ factor.

Within the present scenario, with the latest constraints coming from the LHC $8 \mathrm{TeV}$ results, it is worthwhile to study the current status of the Higgs-radion mixing. As an artifact of the trace anomaly, the production of the radion at the LHC proceeds dominantly through the gluon fusion. The radion can also be produced through vector boson fusion as well as in association with a single massive gauge boson or $t \bar{t}$ pair. Once produced, it can decay to a pair of gluon, photon, or $b \bar{b}$ final states with a sizable cross section. The decay to a pair of massive gauge bosons as well as a pair of Higgs bosons, if kinematically allowed, can also be observed in the LHC. One can observe a light radion at the LHC in the diphoton channel $[42,43]$. In the presence of the Higgs-radion mixing, the coupling of both the scalars to the SM particle is altered. There exists a particular value of mixing, where one of the scalars couples maximally to the gluon and the photon and almost decouples from the massive SM particles. As will be discussed later, the parameter space involving the scenario of the Higgs mixed with the radion mostly is restricted by the current LHC bounds coming from the heavy scalar searches in the $W W$ and the $Z Z$ channel. Therefore, only this particular value of mixing, which can only be probed through the diphoton final state, is still allowed. We first perform a comprehensive analysis of this region of the parameter space (where the radion only couples to the gluon and the photon), for the case of both 13 and $14 \mathrm{TeV}$ LHC, with the radion mass varying from $80 \mathrm{GeV}-1 \mathrm{TeV}$. The $W W$ and $Z Z$ decay modes dominate over most of the mixing parameter space, once kinematically allowed. The hadronic decay channels of these gauge bosons are difficult to observe at the LHC. We therefore repeat our analysis with the radion being produced through gluon fusion and decaying to $Z Z$, with the $Z$ decaying leptonically. We do not consider the $W$ decay channel because the leptonic decay of $W$ is accompanied by missing energy, making it difficult for the scalar mass reconstruction. The mixed radion can also be produced at the LHC via vector boson fusion, but the production rates are suppressed by the vacuum expectation value (VEV) of the radion.

The proposed International Linear Collider (ILC) $[44,45]$ will be the next generation $e^{+} e^{-}$collider, designed to operate at the c.m. energies of 250, 500, and $1000 \mathrm{GeV}$, with integrated luminosity of $250 \mathrm{fb}^{-1}$, $500 \mathrm{fb}^{-1}$, and $1000 \mathrm{fb}^{-1}$, respectively. Compared to the LHC, the leptonic linear collider has moderate hadronic backgrounds and a tunable but restricted center of mass energy. The ILC is mainly intended for precision measurements of the masses and couplings of the SM particles. Therefore, if there is any evidence of new physics at the LHC, a thorough precision study in the ILC will be necessary to pinpoint the validity of the new scenario. The ILC will also have the additional advantage of initial beam polarization, both longitudinal and transverse $\left(60 \%\right.$ for $e^{+}$and $90 \%$ for $\left.e^{-}\right)$. At the ILC, the direct search for the radion $\varphi$ can be via $e^{+} e^{-} \rightarrow Z \varphi$ and $e^{+} e^{-} \rightarrow \nu \bar{\nu} \varphi$, with the radion decaying to either $b \bar{b}$ or $W W / Z Z$. The analysis through these decay channels will help the ILC to probe those regions of the parameter space which will be difficult to explore at the LHC. We have therefore also performed a through analysis, for radion of mass in the range $100 \mathrm{GeV}-1 \mathrm{TeV}$, in the case of the ILC, considering the above production channels. The $b \bar{b}$ and the hadronic decay channel of the $W$ are considered. The main purpose of this work is to show the synergy of the LHC and the ILC in exploring the Higgsradion mixed scenario, in the context of warped model with custodial symmetry.

This paper is organized as follows. In Sec. II, we review the Randall-Sundrum model and the emergence of the radion. The coupling of the Higgs and the radion to the SM particles, prior to mixing, is reviewed in Sec. III, and the mixing case is discussed in Sec. IV. The detailed study in the LHC for the favored parameter space is explored in Sec. V, after accounting for the constraints on the Higgs-radion parameter space, from the latest LHC results in Sec. VA. The complementary study in Sec. VI is devoted to our systematic analysis in the ILC. Finally, we summarize our findings and conclude in Sec. VII.

\section{RANDALL SUNDRUM MODEL}

The original version of the RS model has one extra dimension, compactified on a circle. This compactification has a topology of $S^{1} / \mathcal{Z}_{2}$ orbifold, where $S_{1}$ is a sphere in one dimension and $\mathcal{Z}_{2}$ is the multiplicative group $\{-1,1\}$. By construction, the final picture is of two 3-branes, separated by a distance and enclosing a $5 \mathrm{D}$ bulk. The branes are located at the orbifold fixed points, with $\phi=0$, $\pi$. The two branes are required to have opposite tensions, which cancel the 5D bulk cosmological constant to yield a vanishing four-dimensional (4D) cosmological constant. These 3-branes support the 3+1-dimensional theory and are called the visible (TeV) and the hidden (Planck) branes. The fundamental action describing the above part, excluding the 3-branes, is 


$$
\mathcal{S}=\int d^{4} x \int_{-\pi}^{\pi} d \phi \sqrt{-G}\left(2 M_{5}^{3} \mathcal{R}[G]-\Lambda\right),
$$

where $G$ is the determinant of the five-dimensional metric, $M_{5}$ is the fundamental 5D mass scale, $\mathcal{R}$ is the Ricci scalar, and $\Lambda$ is the bulk cosmological constant. The 5D metric, respecting the four-dimensional Poincare invariance in the $x^{\mu}$ direction, takes the form

$$
d s^{2}=e^{-2 k r_{c}|\phi|} \eta_{\mu \nu} d x^{\mu} d x^{\nu}+r_{c}^{2} d \phi^{2}, \quad-\pi \leq \phi \leq \pi,
$$

with $r_{c}$ the compactification radius and $k$ the bulk curvature. As mentioned before, in the original RS model, the SM particles are localized on the brane. Therefore, the fourdimensional effective action is obtained by integrating out the extra dimension. All the fields have initially masses near the 4D Planck scale, and the fundamental mass parameter is exponentially suppressed, as $M_{\mathrm{TeV}}=e^{-k r_{c} \pi} M_{\text {Planck }}$, with the physical mass being warped down to the weak scale. Since $M_{\mathrm{TeV}} \approx 10^{-16} M_{\text {Planck }}$, the size of the extra dimension is given by $k r_{c} \pi \approx 35$. The compactification radius $r_{c}$ is arbitrary and is treated as a free parameter in the theory. It can be considered as a fluctuation in the extra dimension, which results in the existence of a massless scalar field called the radion. Goldberger and Wise [46,47] proposed an interesting solution to fix the size of extra dimension by introducing a massive scalar field in the bulk with an associated potential, along with interaction terms on the two 3-branes. Taking into account the backreaction of the geometry due to the scalar field, an effective potential is generated, which stabilizes the compactified radius. This effective potential generates mass and VEV of the radion. The 5D metric is then subsequently expanded, taking into account the scalar perturbations $F(x, \phi)$ due to the effect of the radion field,

$d s^{2}=e^{-2\left(k r_{c}|\phi|+F(x, \phi)\right)} \eta_{\mu \nu} d x^{\mu} d x^{\nu}-(1+2 F(x, \phi))^{2} r_{c}^{2} d \phi^{2}$,

where $F(x, \phi)=\varphi(x) R(\phi)$ [48]. Here, $R(\phi)$ is determined by requiring that the metric solves Einstein's equations, whereas $\varphi(x)$ is the canonically normalized 4D scalar field obtained after integrating out the extra dimension. When the backreaction of the metric background due to the scalar field is small, the wave function is then

$$
F(x, \phi)=\frac{\varphi(x)}{\Lambda_{\phi}} e^{2 k r_{c}(\phi-\pi)},
$$

where $\Lambda_{\phi}=\sqrt{6} M_{\text {Planck }} e^{-k r_{c} \pi}$ is the VEV of the radion. The mass of the radion depends on the mass of the bulk scalar and can be smaller than $1 \mathrm{TeV}$. As the other KK fluctuations lie in the scale of at least $2-3 \mathrm{TeV}$, the radion is the lightest scalar in this scenario that can be detected directly at the LHC.

\section{RADION AND HIGGS BOSON COUPLINGS WITH THE SM FERMIONS AND GAUGE BOSONS}

The model considered here is the 5D electroweak group incorporated in a custodial $S U(2)$ symmetry. The gauge group in the bulk is $S U(2)_{L} \times S U(2)_{R} \times U(1)_{B-L}$, with the breaking of $S U(2)_{L} \times S U(2)_{R} \rightarrow S U(2)_{D}$ on the TeV brane, and $S U(2)_{R} \times U(1)_{B-L} \rightarrow U(1)_{Y}$ on the Planck brane. In this picture, the matter and the gauge fields propagate in the bulk. The electroweak symmetry breaking on the $\mathrm{TeV}$ brane is achieved via a localized Higgs as discussed in Ref. [30]. The zero modes of the bulk fields are identified with the SM particles. The fermions are assumed to be localized near the Planck brane, for $c_{L}^{i}>1 / 2, c_{R}^{i}<-1 / 2$, and near the IR brane for $c_{L}^{i}<1 / 2, c_{R}^{i}>-1 / 2$, where $i$ is the flavor index. In order to generate the Yukawa couplings hierarchy, lighter fermions are assumed to be localized near Planck brane, and $t_{R}, t_{L}, b_{L}$ are close to the IR brane. Assuming that the Yukawa coupling matrix is diagonal, the electron Yukawa coupling leads to $c^{e} \simeq 0.64$ and the top Yukawa coupling constrains $c^{t} \simeq-0.5$. Therefore, for the remaining fermions, the corresponding Yukawa couplings are obtained for $c^{t} \simeq c^{i} \simeq c^{e}$ $[49,50]$. In our analysis, the $c^{i}$ for all the fermions except the bottom and the top quark are fixed such that the coupling of the radion to the fermions is similar to the SM Yukawa coupling $\left(c_{L}-c_{R}=1\right)$, that is, with the couplings being proportional to the mass of the fermions. The only difference arises from the fact that the couplings with the radion are suppressed by $\Lambda_{\phi}$ (the radion VEV) instead of $v$ (the Higgs VEV). For the bottom quark, the profile parameters as discussed before are $c_{L}^{b}=0.3$ and $c_{R}^{b}<-0.5$. Similarly for the top quark, we have $c_{L}^{t}=0.3$ and $c_{R}^{t}=0$. The interaction with fermions is then, for the Higgs and the radion,

$$
\begin{gathered}
\mathcal{L}_{h}^{f f}=\frac{h}{v}\left(m_{f} \bar{\psi} \psi\right), \\
\mathcal{L}_{\varphi}^{f f}=\frac{\varphi}{\Lambda_{\phi}}\left(m_{f}\left(c_{L}^{f}-c_{R}^{f}\right) \bar{\psi} \psi\right),
\end{gathered}
$$

where $\left(c_{L}^{f}-c_{R}^{f}\right) \sim 1$ for all the fermions except the $b$ and $t$ quarks.

The radion coupling to the gauge bosons differs from the coupling of the gauge bosons to the Higgs. We first discuss the couplings to massive gauge bosons, where in the case of the SM Higgs the coupling is proportional to the mass. In the case of the radion, due to the propagation of the gauge bosons in the bulk, there is an additional tree level coupling of the radion to the bulk kinetic term of the massive gauge bosons. This additional tree level coupling is also present for the massless gauge bosons propagating in the bulk. The couplings for the Higgs boson and radion with the gauge bosons are, respectively,

$$
\mathcal{L}_{h}^{W W, Z Z}=\frac{h}{v}\left(2 M_{W}^{2} W_{\mu}^{\dagger} W^{\mu}+M_{Z}^{2} Z_{\mu} Z^{\mu}\right),
$$




$$
\begin{aligned}
\mathcal{L}_{\varphi}^{W W, Z Z}= & \frac{\varphi}{\Lambda_{\phi}}\left[2 M_{W}^{2}\left(1-\frac{3 k r_{c} \pi M_{W}^{2}}{\Lambda_{\phi}^{2}}\right) W_{\mu}^{\dagger} W^{\mu}\right. \\
& +M_{Z}^{2}\left(1-\frac{3 k r_{c} \pi M_{Z}^{2}}{\Lambda_{\phi}^{2}}\right) Z_{\mu} Z^{\mu} \\
& \left.+\frac{1}{4 k r_{c} \pi}\left(2 W_{\mu \nu}^{\dagger} W^{\mu \nu}+Z_{\mu \nu} Z^{\mu \nu}\right)\right] .
\end{aligned}
$$

In the case of massless gauge bosons, along with the tree level coupling, the effects of the localized trace anomalies on the $\mathrm{TeV}$ brane are also included, and these are proportional to the $\beta$-function coefficient of the light fields localized on the $\mathrm{TeV}$ brane [48,51]. In addition to these couplings, the SM fermions enter in triangle diagrams involving decays into $\gamma \gamma$ and $g g$ and the $W$ boson in $\gamma \gamma$ diagrams. These triangle diagrams are similar to the massless gauge boson coupling to the Higgs and calculated similarly. There can be additional contributions from the KK fermions and the KK $W$ boson in the triangle loop. We have neglected the contributions of the KK modes in our analysis, ${ }^{1}$ as they are very massive and therefore give negligible contributions. The loop-induced couplings for Higgs and radion are, respectively,

$$
\begin{aligned}
\mathcal{L}_{h}^{g g, \gamma \gamma}=\frac{h}{4 v} & \left(\frac{\alpha_{s}}{2 \pi} b_{\mathrm{QCD}}^{h} G_{\mu \nu}^{a} G^{a \mu \nu}+\frac{\alpha}{2 \pi} b_{\mathrm{EM}}^{h} F_{\mu \nu} F^{\mu \nu}\right), \\
\mathcal{L}_{\varphi}^{g g, \gamma \gamma}= & \frac{\varphi}{4 \Lambda_{\phi}}\left[\left(\frac{1}{k r_{c} \pi}+\frac{\alpha_{s}}{2 \pi} b_{\mathrm{QCD}}^{R}\right) G_{\mu \nu}^{a} G^{a \mu \nu}\right. \\
& \left.+\left(\frac{1}{k r_{c} \pi}+\frac{\alpha}{2 \pi} b_{\mathrm{EM}}^{R}\right) F_{\mu \nu} F^{\mu \nu}\right]
\end{aligned}
$$

where $\alpha_{s}$ and $\alpha$ are the strong and the electromagnetic coupling constants and we define

$$
\begin{gathered}
b_{\mathrm{QCD}}^{h}=\tau_{f}\left(1+\left(1-\tau_{f}\right) f\left(\tau_{f}\right)\right), \\
b_{\mathrm{EM}}^{h}=\frac{8}{3}\left[\tau_{f}\left(1+\left(1-\tau_{f}\right) f\left(\tau_{f}\right)\right)\right] \\
-\left[2+3 \tau_{W}+3 \tau_{W}\left(2-\tau_{W}\right) f\left(\tau_{W}\right)\right], \\
b_{\mathrm{QCD}}^{R}=7+\tau_{f}\left(1+\left(1-\tau_{f}\right) f\left(\tau_{f}\right)\right),
\end{gathered}
$$

\footnotetext{
${ }^{1}$ The contributions of the KK modes for $W$ and $f$ depend on the cutoff scale $\Lambda_{\phi}$, and additionally $K K f$ depend on the fermion bulk mass parameter. The contributions of KK $W$ bosons and fermions amount to 0.06 the SM one and $K K t$ being 0.012 the $\mathrm{SM}$ one, for $\Lambda_{\phi}=4 \mathrm{TeV}$ [17]. The $K K W$ contributions to $g g$ amount to $<5 \%$ [52]. These contributions are suppressed with respect to the trace anomaly part, and hence we have ignored them.
}

$$
\begin{aligned}
b_{\mathrm{EM}}^{R}= & -\frac{11}{3}+\frac{8}{3}\left[\tau_{f}\left(1+\left(1-\tau_{f}\right) f\left(\tau_{f}\right)\right)\right] \\
& -\left[2+3 \tau_{W}+3 \tau_{W}\left(2-\tau_{W}\right) f\left(\tau_{W}\right)\right], \\
f(\tau)= & \left(\sin ^{-1} \frac{1}{\sqrt{\tau}}\right)^{2} \quad(\text { for } \tau>1) \quad \text { and } \\
- & \frac{1}{4}\left(\log \frac{\eta_{+}}{\eta_{-}}-i \pi\right)^{2} \quad(\text { for } \tau<1) \\
\eta_{ \pm}= & 1 \pm \sqrt{1-\tau}, \quad \tau_{i}=\left(\frac{2 m_{i}}{m_{S}}\right)^{2} .
\end{aligned}
$$

In Eq. (16), $m_{i}$ is the mass of the particle in the loop, and $m_{S}$ is either the mass of the radion or the Higgs, depending on the coupling. The $\tau_{f}=m_{f}^{2} / m_{S}^{2}$ in the above equations denotes fermion mass ratios squared, whereas $\tau_{W}=$ $m_{W}^{2} / m_{S}^{2}$ is for the $W$ gauge boson. Through its interaction with the stress-energy momentum tensor, the radion couples with the Higgs boson as

$$
\mathcal{L}_{\varphi}^{h h}=\frac{\varphi}{\Lambda_{\phi}}\left(-\partial_{\mu} h \partial^{\mu} h+2 m_{h}^{2} h^{2}\right)
$$

\section{HIGGS-RADION MIXING}

The operator giving rise to mixing between the radion and the Higgs boson follows from the principle of general covariance [10]:

$$
S_{\xi}=\xi \int d^{4} x \sqrt{g_{\text {vis }}} \mathcal{R}\left(g_{\text {vis }}\right) \hat{H}^{\dagger} \hat{H}
$$

Here, $\xi$ is the mixing parameter, $g_{\text {vis }}^{\mu \nu}$ is the metric induced on the visible brane, and $\mathcal{R}\left(g_{\text {vis }}\right)$ is a four-dimensional Ricci scalar of the induced metric. After expanding the radion field about its $\mathrm{VEV}$ and keeping only the terms containing bilinear fields, we get

$$
\begin{aligned}
\mathcal{L}_{\text {mix }}= & -\frac{1}{2}\left(1+6 \gamma^{2} \xi\right) \varphi \square \varphi-\frac{1}{2} \varphi m_{\varphi}^{2} \varphi \\
& -\frac{h}{2}\left(\square+m_{h}^{2}\right) h-6 \xi \gamma h \square \varphi,
\end{aligned}
$$

where $m_{\varphi}$ and $m_{h}$ are the radion and the Higgs mass. In order to diagonalize the kinetic part of $\mathcal{L}_{\text {mix }}$, we consider the following transformation,

$$
\begin{aligned}
h & =\left(\cos \theta+\frac{6 \xi \gamma}{Z} \sin \theta\right) h_{m}+\left(\sin \theta-\frac{6 \xi \gamma}{Z} \cos \theta\right) r_{m} \\
& =a_{\theta} h_{m}+b_{\theta} r_{m}, \\
\varphi & =\frac{-\sin \theta}{Z} h_{m}+\frac{\cos \theta}{Z} r_{m}=c_{\theta} h_{m}+d_{\theta} r_{m},
\end{aligned}
$$

where $Z^{2}=1+6 \xi \gamma^{2}-36 \xi^{2} \gamma^{2}$ and the mixing angle is 

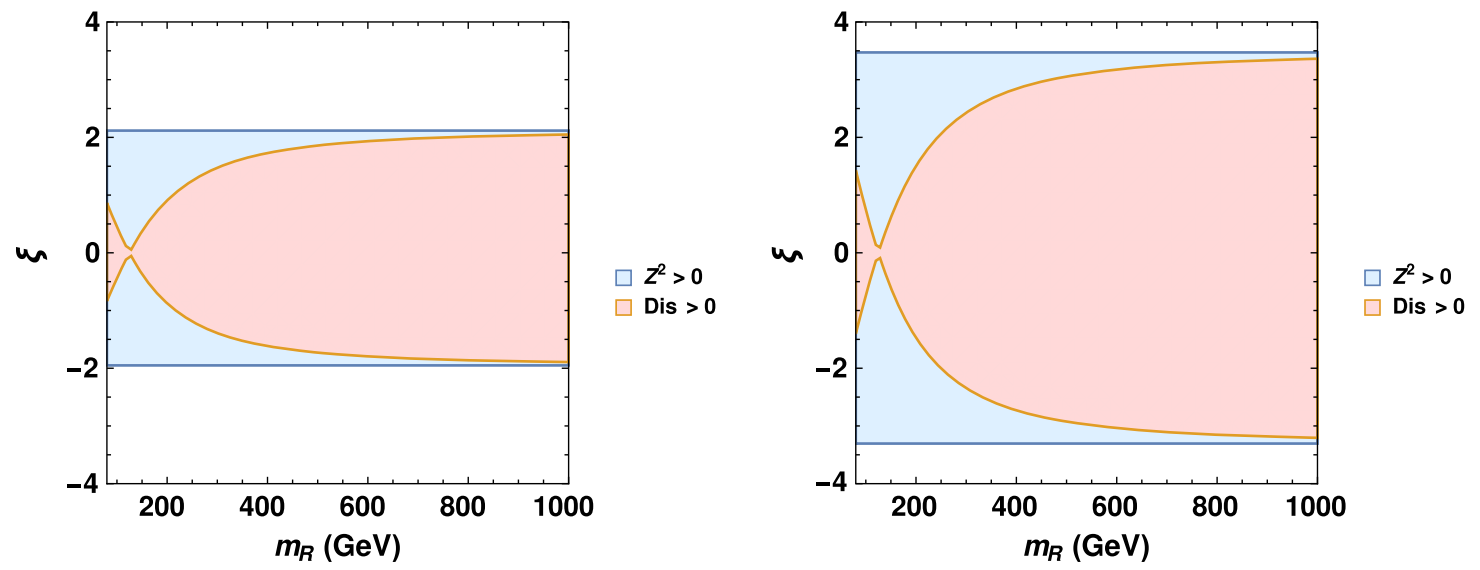

FIG. 1. The allowed parameter region for $\xi$ satisfying Eq. (24) (in blue for $Z^{2}>0$, in pink for Dis $>0$ ), as a function of the radion mass $m_{R}$. We consider $\Lambda_{\phi}=3 \mathrm{TeV}$ (left panel) and $5 \mathrm{TeV}$ (right panel) and take $m_{H}=125 \mathrm{GeV}$.

$$
\tan 2 \theta=\frac{12 \xi \gamma Z m_{h}^{2}}{\left[m_{h}^{2}\left(Z^{2}-36 \xi^{2} \gamma^{2}\right)-m_{\varphi}^{2}\right]}
$$

The corresponding mass eigenvalues of the physical fields $\left[h_{m}\left(r_{m}\right)\right]$ are then given by

$m_{ \pm}^{2}=\frac{1}{2 Z^{2}}\left[m_{\varphi}^{2}+\beta m_{h}^{2} \pm \sqrt{\left(m_{\varphi}^{2}+\beta m_{h}^{2}\right)^{2}-4 Z^{2} m_{h}^{2} m_{\varphi}^{2}}\right]$

where $\beta=1+6 \gamma^{2} \xi$ and we denote the larger of $\left[m_{h_{m}}, m_{r_{m}}\right]$ with $m_{+}$. Equation (22) can be inverted to express $\left(m_{h}, m_{\varphi}\right)$ in terms of $m_{ \pm}$:

$\left[\beta m_{h}^{2}, m_{\varphi}^{2}\right]=\frac{Z^{2}}{2}\left[m_{+}^{2}+m_{-}^{2} \pm \sqrt{\left(m_{+}^{2}+m_{-}^{2}\right)^{2}-\frac{4 \beta m_{+}^{2} m_{-}^{2}}{Z^{2}}}\right]$.

Thus, the Higgs-radion mixed system can be described by four independent parameters, i.e. the mixing parameter $\xi$, the radion $\mathrm{VEV} \Lambda_{\phi}$, and the physical masses of the two mixed scalars $\left(m_{h_{m}}\right)$ and $\left(m_{r_{m}}\right)$. In order to remove the quadratic ambiguity in the equation for physical masses, we adopt the convention where $H$ is the Higgs-like and $R$ is the radionlike scalar. ${ }^{2}$ From now on, we will refer to the scalars $h_{m}$ and $r_{m}$ as $H$ and $R$, and similarly their masses will be denoted by $m_{H}$ and $m_{R}$, respectively. Moreover, for given values of physical states masses $m_{H}$ and $m_{R}$, there is an additional constraint on the mixing parameter $\xi$, obtained by demanding that the quantity inside the square root of Eq. (23) be positive. Therefore, the parameter $\xi$ must satisfy

\footnotetext{
${ }^{2}$ Higgs-like, because it behaves like the SM Higgs boson in the limit $\xi \rightarrow 0$.
}

$$
\left(m_{H}^{2}+m_{R}^{2}\right)^{2}-\frac{4 \beta m_{H}^{2} m_{R}^{2}}{Z^{2}}>0 \text { and } Z^{2}>0 .
$$

We assume that the Higgs-like mixed scalar has mass $m_{H}=125 \mathrm{GeV}$, and the radionlike mixed scalar $R$ should satisfy the current experimental limits (to be discussed later). In Fig. 1, we plot the allowed range of $\xi$ as a function of $m_{R}$, for $\Lambda_{\phi}=3$ and $5 \mathrm{TeV}$ satisfying Eq. (24), with $m_{H}$ fixed to $125 \mathrm{GeV}$. The expression Dis in Fig. 1 refers to $\left(m_{H}^{2}+m_{R}^{2}\right)^{2}-\left(4 \beta m_{H}^{2} m_{R}^{2}\right) / Z^{2}$ in Eq. (24). The area enclosed by the blue region has $Z^{2}>0$, and the red region shows the allowed parameter region with Dis $>0$. The theoretically allowed parameter space increases with $\Lambda_{\phi}$ as can be seen in Fig. 1.

\section{A. Decays of the mixed scalars $(H, R)$ to $\mathrm{SM}$ particles}

Below, we list the interactions of the mixed scalars with the SM particles. The expressions can be easily obtained using Eq. (20), for the interactions listed in the previous section, Sec. III. From this point onward, we will refer to the mixed Higgs $H$ as the Higgs and mixed radion $R$ as the radion. The effective Lagrangians for the decay of the Higgs and radion into a pair of massive gauge boson and into fermion pairs are given by, respectively,

$$
\begin{aligned}
\mathcal{L}_{H}^{W W, Z Z}= & \frac{H}{v}\left\{\left[a_{\theta}+\gamma c_{\theta}\left(1-\frac{3 k r_{c} \pi M_{W}^{2}}{\Lambda_{\phi}^{2}}\right)\right] 2 M_{W}^{2} W_{\mu}^{\dagger} W^{\mu}\right. \\
& +\frac{\gamma c_{\theta}}{2 k r_{c} \pi} W_{\mu \nu}^{\dagger} W^{\mu \nu}+\left[a_{\theta}+\gamma c_{\theta}\left(1-\frac{3 k r_{c} \pi M_{Z}^{2}}{\Lambda_{\phi}^{2}}\right)\right] \\
& \left.\times M_{Z}^{2} Z_{\mu} Z^{\mu}+\frac{\gamma c_{\theta}}{4 k r_{c} \pi} Z_{\mu \nu} Z^{\mu \nu}\right\}
\end{aligned}
$$



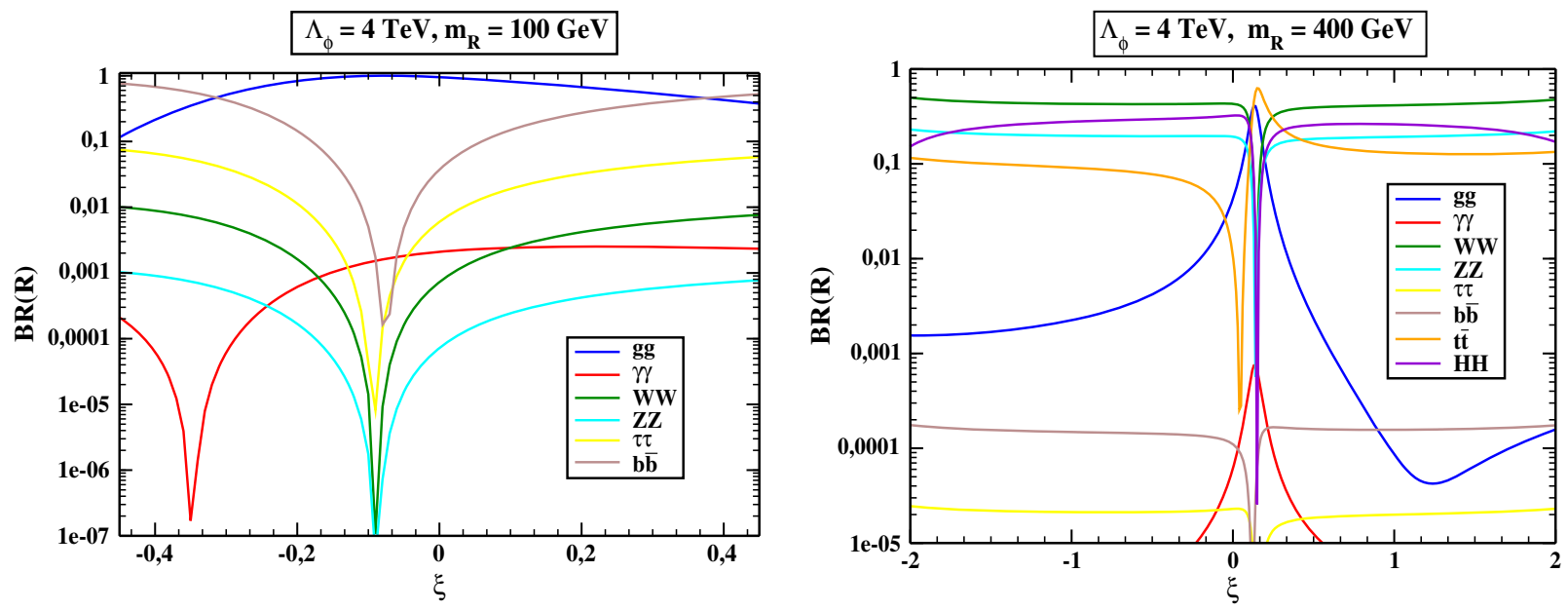

FIG. 2. The branching ratio of the mixed radion state to kinematically allowed final states as a function of mixing parameter $(\xi)$, for $\Lambda_{\phi}=4 \mathrm{TeV}$, and for $m_{R}=100 \mathrm{GeV}$ (left panel) and $400 \mathrm{GeV}$ (right panel).

$$
\begin{aligned}
\mathcal{L}_{R}^{W W, Z Z}= & \frac{R}{v}\left\{\left[b_{\theta}+\gamma d_{\theta}\left(1-\frac{3 k r_{c} \pi M_{W}^{2}}{\Lambda_{\phi}^{2}}\right)\right] 2 M_{W}^{2} W_{\mu}^{\dagger} W^{\mu}\right. \\
& +\frac{\gamma d_{\theta}}{2 k r_{c} \pi} W_{\mu \nu}^{\dagger} W^{\mu \nu} \\
& +\left[b_{\theta}+\gamma d_{\theta}\left(1-\frac{3 k r_{c} \pi M_{Z}^{2}}{\Lambda_{\phi}^{2}}\right)\right] M_{Z}^{2} Z_{\mu} Z^{\mu} \\
& \left.+\frac{\gamma d_{\theta}}{4 k r_{c} \pi} Z_{\mu \nu} Z^{\mu \nu}\right\} \\
\mathcal{L}_{H}^{f f} & =\frac{H}{v}\left[a_{\theta}+\gamma c_{\theta} \frac{1}{2}\left(c_{L}-c_{R}\right)\right]\left(m_{f} \bar{\psi} \psi\right) \\
\mathcal{L}_{R}^{f f} & =\frac{R}{v}\left[b_{\theta}+\gamma d_{\theta} \frac{1}{2}\left(c_{L}-c_{R}\right)\right]\left(m_{f} \bar{\psi} \psi\right)
\end{aligned}
$$

Similarly, the interaction terms of the radion and the Higgs with the massless gauge bosons in the mixing scenario are given by

$$
\begin{aligned}
\mathcal{L}_{H}^{g g, \gamma \gamma}= & \frac{H}{4 v}\left\{\left[a_{\theta} \frac{\alpha_{s}}{2 \pi} b_{\mathrm{QCD}}^{h}+\gamma c_{\theta}\left(\frac{1}{k r_{c} \pi}+\frac{\alpha_{s}}{2 \pi} b_{\mathrm{QCD}}^{R}\right)\right] G_{\mu \nu}^{a} G^{a \mu \nu}\right. \\
& \left.+\left[a_{\theta} \frac{\alpha}{2 \pi} b_{\mathrm{EM}}^{h}+\gamma c_{\theta}\left(\frac{1}{k r_{c} \pi}+\frac{\alpha}{2 \pi} b_{\mathrm{EM}}^{R}\right)\right] F_{\mu \nu} F^{\mu \nu}\right\}, \\
\mathcal{L}_{R}^{g g, \gamma \gamma}= & \frac{R}{4 v}\left\{\left[b_{\theta} \frac{\alpha_{s}}{2 \pi} b_{\mathrm{QCD}}^{h}+\gamma d_{\theta}\left(\frac{1}{k r_{c} \pi}+\frac{\alpha_{s}}{2 \pi} b_{\mathrm{QCD}}^{R}\right)\right] G_{\mu \nu}^{a} G^{a \mu \nu}\right. \\
& \left.+\left[b_{\theta} \frac{\alpha}{2 \pi} b_{\mathrm{EM}}^{h}+\gamma d_{\theta}\left(\frac{1}{k r_{c} \pi}+\frac{\alpha}{2 \pi} b_{\mathrm{EM}}^{R}\right)\right] F_{\mu \nu} F^{\mu \nu}\right\}
\end{aligned}
$$

where $a_{\theta}, b_{\theta}, c_{\theta}$, and $d_{\theta}$ are defined in Eq. (20) and $\gamma=v / \Lambda_{\phi}$. Finally, when $m_{R}>2 m_{H}$, the heavier scalar can decay into a pair of lighter scalars. The coupling between the two scalars in the mixed scenario comes from three basic sources: $(a)$ the interaction of the radion with the trace of the SM Higgs field, also present in the unmixed case Eq. (17); (b) the trilinear term in the Higgs potential; and (c) a contribution from the Higgs-radion mixing term in Eq. (18). The interaction Lagrangian of the radion with two Higgs bosons is therefore given by

$$
\begin{aligned}
\mathcal{L}_{R}^{H H}= & \frac{R}{\Lambda_{\phi}}\left\{\left[-a_{\theta}^{2} d_{\theta}\left(\partial_{\mu} H \partial^{\mu} H+2 m_{H}^{2} H H\right)\right]\right. \\
& \left.+4 a_{\theta} b_{\theta} c_{\theta} m_{H}^{2} H H\right\}-3 \frac{m_{H}^{2}}{2 v}\left(a_{\theta}^{2} b_{\theta} R H^{2}\right) \\
& -\frac{3 \xi}{\Lambda_{\phi}}\left[\left(a_{\theta}^{2} d_{\theta} H^{2} \square R+2 a_{\theta} b_{\theta} c_{\theta} H \square H R\right)\right] \\
& -6 \xi \frac{v}{\Lambda_{\phi}}\left[\left(a_{\theta} c_{\theta} d_{\theta} H^{2} \square R+\left(a_{\theta} d_{\theta}+b_{\theta} c_{\theta}\right) c_{\theta} H \square H R\right)\right] .
\end{aligned}
$$

We plot the branching ratios of the radion to different allowed final states in Fig. 2, as a function of the mixing parameter, $\xi$, for two radion masses, 100 and $400 \mathrm{GeV}$. We can see from Fig. 2 that for a certain value of $\xi\left(\xi<0\right.$ for $m_{R}=100 \mathrm{GeV}$, $\xi>0$ for $m_{R}=400 \mathrm{GeV}$ ), the decays of the radion $R$ to the SM leptons, quarks, and massive gauge bosons are suppressed. At this point, in the expression appearing in Eqs. (26) and (28) $b_{\theta}+\gamma d_{\theta} \sim 0$, whereas the radion couplings to $\gamma \gamma$ and $g g$ have extra contributions coming from trace anomaly and bulk kinetic term, and hence these are the dominant decay modes. Due to propagation in the bulk, the $W W$ and $Z Z$ radion couplings also have additional small terms, but their effect is very small and can be ignored. Hence, we divide our analysis into two regions:

(i) Region 1 (the LHC region). - In this region, the radion is coupled maximally to the massless gauge bosons. This region of parameter space is ideally suited for study at the LHC, where we can focus on 
the production of the mixed scalar via gluon fusion production and its subsequent decay to a diphoton.

(ii) Region 2 (the ILC region).-A Although this region of parameter space can be studied in the LHC with the radion being produced through the vector boson fusion followed by decay to massive vector bosons, the production cross sections for the associate channels are suppressed by the VEV of the radion, $\Lambda_{\phi}$. We considered the gluon fusion production of the heavy scalar and its decay to vector bosons. This region of parameter space can also be probed by the associated production of the heavy scalar and the top or massive vector bosons and its decay to $b \bar{b}$. But the $b \bar{b}$ final states are mostly accompanied by large hadronic background in the LHC, and this is difficult to probe. Thus, this scenario is better suited for study at the ILC. We concentrate on the production of the radion by its associated production with $Z$ and the vector boson fusion, followed by and its decay to $b \bar{b}$ and massive gauge bosons, depending on the mass of the radion.

We will discuss these regions in detail in two sections, Secs. V and VI.

\section{PROSPECTS FOR THE SEARCHES AT THE 13 AND 14 TEV LHC}

In this section, we first discuss the present limits derived on the mass of the radion and its mixing parameter $\xi$, then incorporate these into an investigation of the parameter space which can be probed at the LHC at $\sqrt{s}=13$ and $14 \mathrm{TeV}$.

\section{A. Constraints on the $\xi$ and $m_{R}$ values from the LHC data}

During run 1, the LHC experiment has looked for additional scalar particles, other than the Higgs boson, decaying through narrow resonances into different final states. We assumed the scenario where the mixed-Higgs scalar $(H)$ mimics the scalar at $125 \mathrm{GeV}$ discovered in LHC run 1 . The nonobservation of the other scalar in LHC run 1 puts constraints on the values of $\Lambda_{\phi}$ and $\xi$. We show our results for $m_{R}$ in the mass range of $80 \mathrm{GeV}$ to $1 \mathrm{TeV}$ in Fig. 3. The experimental data used to constrain the $\xi-m_{R}$ parameter space come from the diphoton [53-55], $W W$ [56,57], ZZ [58], $b \bar{b}$ [59], and $\tau^{+} \tau^{-}$[60] searches at the LHC. When the mass of the radion is greater than $250 \mathrm{GeV}$, the heavy scalar can decay into a pair of SM Higgs bosons. We have included the bounds coming from the LHC searches for Higgs boson pair production, in the $4 b$ final state $[61,62]$. We scanned the $\xi-m_{R}$ parameter space for $\Lambda_{\phi}=3,4$ and $5 \mathrm{TeV},{ }^{3}$ taking into account all of the above

\footnotetext{
${ }^{3}$ We started with $\Lambda_{\phi}>2.5 \mathrm{TeV}$, which is compatible with the minimum bound on the mass of the spin- $1 \mathrm{KK}$ resonance.
}

experimental results, and also imposed the theoretical bound, stated in Eq. (24). The LHC search channels, which constrain the $\xi-m_{R}$ parameter space, are more restrictive than the theoretical bound, as shown in Fig. 3, where the allowed parameter space is plotted in the $\xi-m_{R}$ plane, for different $\Lambda_{\phi}$, for both heavy and light states. The parameter space is mostly constrained by the heavy Higgs data from the $W W$ (region in pink) and $Z Z$ (region in pink + gray) final state. When $m_{R}<125 \mathrm{GeV}$, the parameter space is mostly excluded by diphoton searches (region in green) and from Higgs signal strength measurement, while for $m_{R}>250 \mathrm{GeV}$, the decay into two Higgs bosons ( $4 b$ final states) restricts the parameter space further (region in orange). ${ }^{4}$ Finally, the region in blue represents the theoretical restrictions on the parameter space.

The white region in Fig. 3 is the one currently allowed by the LHC experiments and the theory. We highlight this region in Fig. 4 for $\Lambda_{\phi}=3,4$ and $5 \mathrm{TeV}$. In these plots, apart from the LHC heavy Higgs data mentioned above, we have also included the constraints from the Higgs signal strength $8 \mathrm{TeV}$ LHC data [63] as well as the constraints from the LHC $13 \mathrm{TeV}$ data, showing an excess of $3.9 \sigma$ (ATLAS [64]) and 2.6 $\sigma$ (CMS [65]) in the diphoton final state at $m_{\gamma \gamma}=750 \mathrm{GeV}$. We find that the negative $\xi$ region is ruled out by the LHC data for $m_{R}>125 \mathrm{GeV}$. The region in brown is the theoretically allowed one, whereas the regions surviving after taking into account the additional constraints for each scale are shown in green for $\Lambda_{\phi}=5 \mathrm{TeV}$, in blue for $\Lambda_{\phi}=4 \mathrm{TeV}$, and in yellow for $\Lambda_{\phi}=3 \mathrm{TeV}$. The values for different $\Lambda_{\phi}$ are superimposed; i.e. for example, for $\Lambda_{\phi}=5 \mathrm{TeV}$, the parameter space allowed covers the regions in green, blue, and yellow; for $\Lambda_{\phi}=4 \mathrm{TeV}$, the allowed parameter space covers the regions in blue and yellow; whereas for $\Lambda_{\phi}=3 \mathrm{TeV}$, the region in yellow is the allowed one. We also find that there is a narrow region about $\xi \sim 0.1(-0.1)$ for $m_{R}>200 \mathrm{GeV}$ $\left(m_{R}<100 \mathrm{GeV}\right)$, which is allowed for almost all mass ranges. This region corresponds to $b_{\theta}+\gamma d_{\theta} \sim 0$ and can only be probed through the diphoton channel at the LHC.

\section{B. Signal analysis for search at the LHC}

We performed our analysis for the LHC, with the mass of the radion varied from $80 \mathrm{GeV}$ to $1 \mathrm{TeV}$. The radion can be produced via gluon fusion, vector boson fusion, and associated production, with the top or the vector bosons produced along with the radion. The production mechanism of the radion is similar to that of the SM Higgs bosons. As discussed before, compared to the Higgs, in the case of the radion the $\gamma \gamma$ and $g g$ decay channels are enhanced from the trace anomaly. One of the unique

\footnotetext{
${ }^{4}$ Please note that the jagged edges of Fig. 3 originate from the exclusion plot of ATLAS and CMS heavy Higgs searches in the $h h \rightarrow 4 b$ channel and do not disappear with increasing the number plot points.
} 

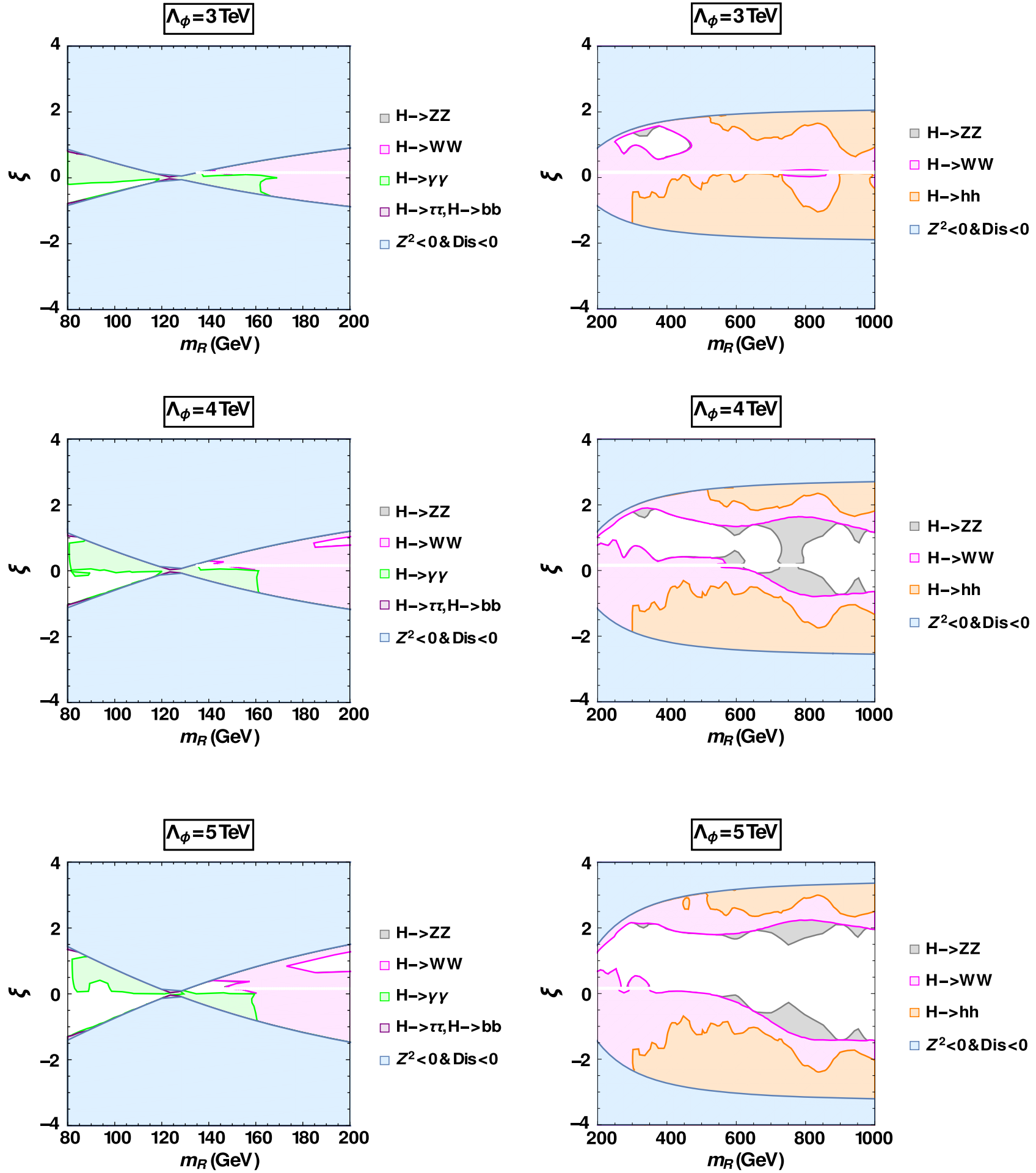

FIG. 3. The exclusion plot in the $\xi-m_{R}$ plane for $\Lambda_{\phi}=3,4$, and $5 \mathrm{TeV}$ from additional scalar $(H)$ searches in the $8 \mathrm{TeV}$ LHC for different decay channels for (left panel) light states and (right panel) heavy states. Here, $H$ states represent the additional scalar. We show the theoretical restrictions (in blue), the additional restrictions from decays into $W^{+} W^{-}$(in pink), $Z Z$ (in gray), $\tau^{+} \tau^{-}$and $b \bar{b}$ (in purple), $h h$ (in orange), and $\gamma \gamma$ (in green). The region in white is allowed as surviving all restrictions. 

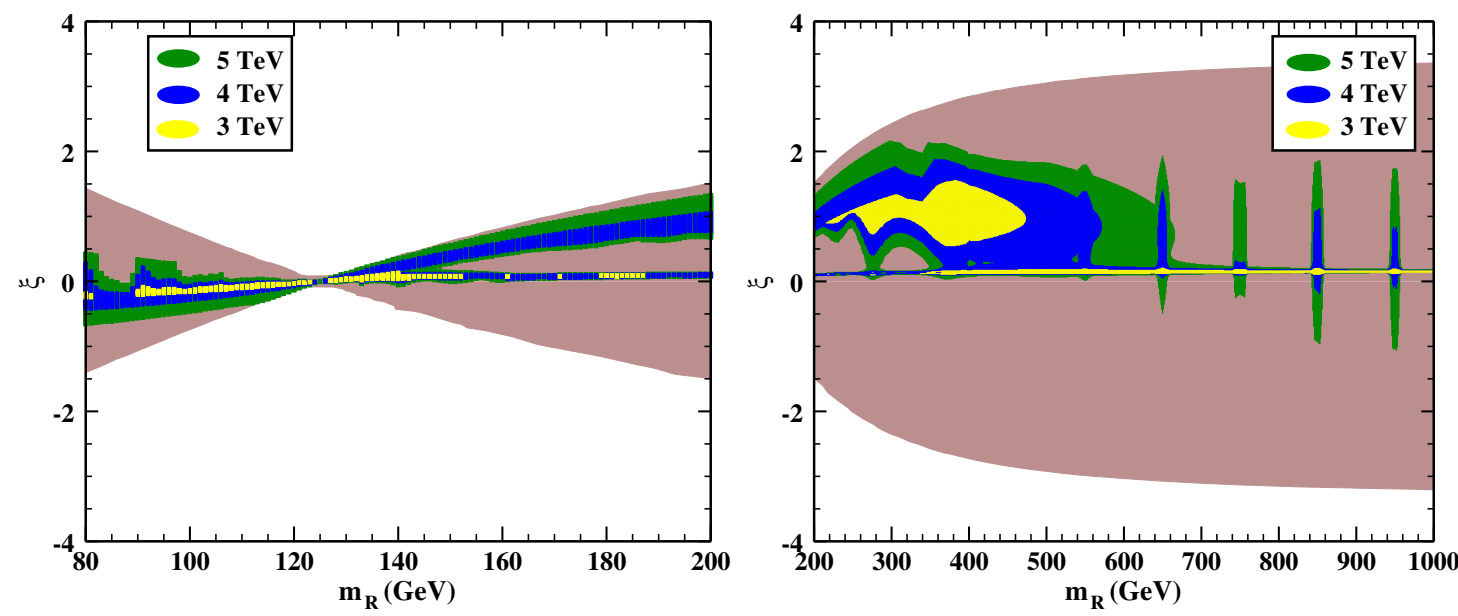

FIG. 4. The allowed parameter space in the $\xi-m_{R}$ plane for $\Lambda_{\phi}=3,4$, and $5 \mathrm{TeV}$ for (left panel) light states and (right panel) heavy states. The region in brown is the theoretically allowed one, while yellow is the allowed region for $\Lambda_{\phi}=3 \mathrm{TeV}$; yellow and blue for $\Lambda_{\phi}=4 \mathrm{TeV}$; and green, in addition to the previous colors, for $\Lambda_{\phi}=5 \mathrm{TeV}$.

features of the Higgs-radion mixing is that there exists a particular value of mixing still allowed by LHC run 1 , where the radion can decay only to a pair of photons or to a pair of gluons, referred to as region 1 in our analysis. ${ }^{5}$ As the diphoton final states are very clean to reconstruct at the LHC, we first perform our analysis through this channel. The $\xi$ parameter space has regions where the coupling to the massive gauge bosons is maximal, leading to the $W W$ and $Z Z$ decay mode. The $W W$ and the $Z Z$ will either decay leptonically or hadronically. The leptonic final states of $W$ are accompanied by missing energy, and hence mass reconstruction is not possible. Thus, this channel cannot be used as the channel for discovery. On the other hand, the leptonic final state of $Z Z$ can be used for mass reconstruction. However, the cross section of the production of the radion via vector boson fusion and its decay to four leptons mediated by $Z$ is suppressed by the VEV of the radion, $\Lambda_{\phi}$. We therefore consider the production of the radion through gluon fusion and its decay to four leptons mediated by $Z$.

\section{Analysis in the $g g \rightarrow R \rightarrow \gamma \gamma$ decay channel}

We first focus on the diphoton channel at the LHC, since this is the cleanest signal. We consider two isolated photons as our signal. The final state with two isolated photons can be mimicked by several SM processes:

(i) Irreducible diphoton background: This background is produced from two prompt photons coming from tree level $q \bar{q}$ annihilation as well as gluon-gluon box

\footnotetext{
${ }^{5}$ Several analyses [66-68] were performed, which use this particular mixing value to explain the $750 \mathrm{GeV}$ diphoton excess. Here, $\Lambda_{\phi}>2.5 \mathrm{TeV}$, and therefore, for this case, the cross section times decay rates are much lower than the observed value of $5-15 \mathrm{fb}$.
}

diagram with quarks propagating in the loop. The production rate from the box diagram is comparable to that from the tree level process, due to a high gluon flux at small $x$, where $x$ is the energy fraction carried by each parton. These photons are mostly isolated.

(ii) Reducible background: There are three backgrounds in this category:

(a) $j \gamma$ background: The $\pi^{0}, \eta$, and $\rho$ inside a jet can decay into two collimated photons which can be identified as a single photon candidate in an electromagnetic calorimeter. However, the photons are mostly nonisolated and hence can be suppressed by using photon isolation criteria.

(b) $j j$ background: Similar to $j \gamma$, each of the jets can produce two collimated photons and hence can mimic two photon states. However, this background can also be completely removed by photon isolation.

(c) Drell-Yan background: The electrons can fake a photon when the tracks of the electrons are not properly reconstructed by inner tracking chamber. We considered the Drell-Yan background with a track mismeasurement efficiency of about 5\%. This background is comparable to the irreducible background near the $Z$ mass.

The signal and the background events with showering and hadronization are generated at the leading order in PYTHIA 8 [69]. We have used CTEQ611 [70] as our parton density function (PDF). The renormalization and factorization scales for both the signal and the background are kept at their default values. In order to enhance the statistics for the signal over the background events, we divided our analysis into different phase space regions, depending upon the mass of the radion. We classified different regions of $\hat{m}$ (the invariant 

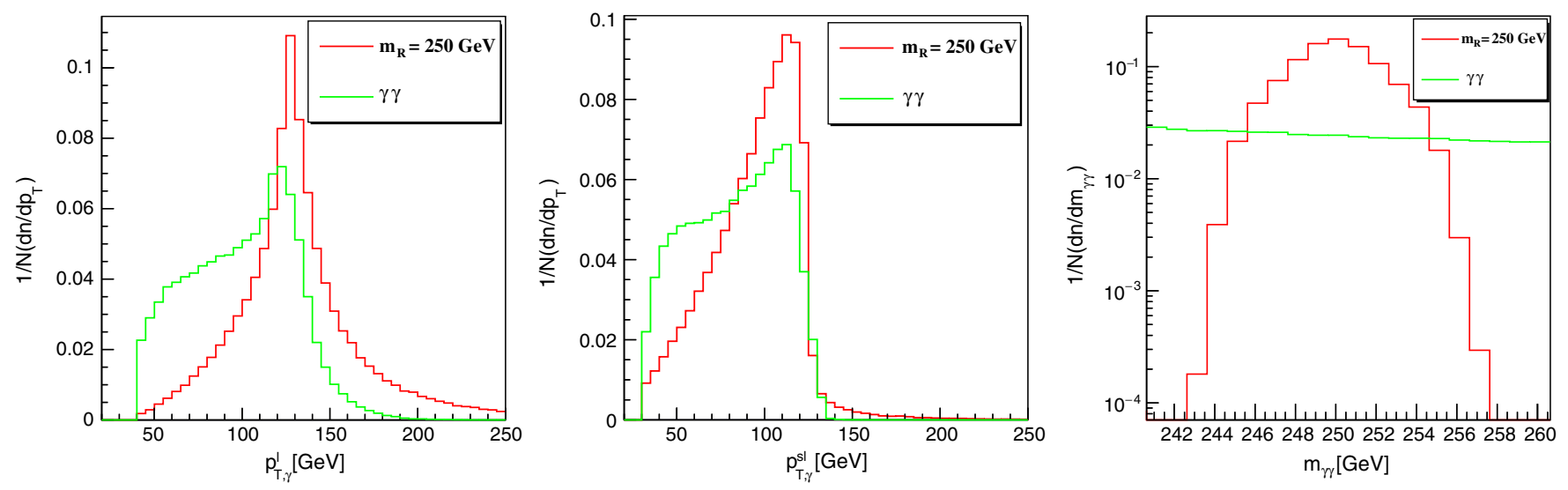

FIG. 5. The $p_{T}$ distribution of the leading $p_{T}^{l}$ (left panel) and subleading photon $p_{T}^{s l}$ (middle panel) and the invariant mass distribution of the (leading + subleading) photons (right panel), for a radion with mass of $250 \mathrm{GeV}$. The red line represents the signal, and the green line represents the $(\gamma \gamma)$ background.

mass of the outgoing partons) depending on the mass of the radion. ${ }^{6}$ In order to have robust signal-background estimations, we implemented an algorithm which approximates the clustering procedure in an electromagnetic calorimeter. The electromagnetic shower from an unconverted photon is contained within a $5 \times 5$ crystal matrix around the seed (the actual hit spot). For a converted photon, the region is even wider. We considered a cluster of such hits within a cone of $\Delta R=0.09$. The momentum of the photon candidate is defined as the vector sum of all the photons and the electrons within the cone of $\Delta \mathrm{R}$ of 0.09 around the seed, which is the direct photon or electron. To account for the detector resolution, we smeared photons, electrons, and jets with Gaussian functions. We selected photon candidates with $p_{T}^{\text {leading(subleading) }}>30(25) \mathrm{GeV}$ and lying within $|\eta|<2.5$, while considering signals for $m_{R}<200 \mathrm{GeV}$. For $m_{R}>200 \mathrm{GeV}$, we selected photon candidates with $p_{T}^{\text {leading(subleading) }}>40(30) \mathrm{GeV}$. Jets are reconstructed with $|\eta|<4.7$ and $p_{T}>25(20) \mathrm{GeV}$. Photons with $1.44<$ $|\eta|<1.55$ are not considered. The triggered photons are required to have minimal hadronic activity. This has been ensured by demanding that the total scalar sum of the transverse energy within the cone of $\Delta R=0.3$ should be less than $5 \mathrm{GeV}$. We further demand that the two photon candidates should be separated by at least $\Delta R=0.4$. Surviving events with two such "isolated" photons qualify for our further analysis. ${ }^{7}$ We found that $j j$ background is completely removed by demanding two such isolated photons. The $p_{T}$ distribution of the photons coming from the background peaks below the signal. Also, as $p_{T}$ of the photon increases, the misidentification rate for $\gamma-j$ decreases. Thus, the background can be separated from the signal by applying

\footnotetext{
${ }^{6}$ We imposed $\left|\hat{m}-m_{R}\right|<15 \mathrm{GeV}$.

${ }^{7}$ When mass of the radion is less than $200 \mathrm{GeV}$, we found that absolute isolation works better; however, beyond $200 \mathrm{GeV}$, both the isolation criteria have almost the same efficiency.
}

a relatively harder $p_{T}$ cut. After applying the $p_{T}$ cut on the leading $(l)$ and subleading $(s l)$ photons, we finally select only those events that lie within the $10 \mathrm{GeV}$ invariant mass bin about the radion mass. The kinematic cuts considered for this analysis are

$$
\begin{aligned}
p_{T}^{l} & >0.5 m_{R}+5.0 \quad \text { and } \quad p_{T}^{s l}>p_{T}^{l}-5.0, \\
\left|m_{\gamma \gamma}-m_{R}\right| & <3.0 \quad \text { when } m_{R} \leq 200 \mathrm{GeV}
\end{aligned}
$$

and

$$
\begin{aligned}
p_{T}^{l} & >0.5 m_{R}-10.0 \quad \text { and } \quad p_{T}^{s l}>p_{T}^{l}-5.0, \\
\left|m_{\gamma \gamma}-m_{R}\right| & <5.0 \quad \text { when } m_{R}>200 \mathrm{GeV} .
\end{aligned}
$$

The hard $p_{T}$ cut on the leading as well as the subleading photons helps to get rid of $\gamma-j$ background, in the low mass region. Although we lose some signal events from this cut, the significance increases appreciably. The gluon fusion cross section decreases with the increase in mass of the radion, and hence we applied a relatively softer $p_{T}$ cut on the photons. As the production rate for diphoton background falls with $p_{T}$ of the photon, we still achieve higher significance, as seen in Fig. 5, where we plot the normalized $p_{T}$ distribution of the leading and subleading photons, as well as the invariant mass for the (leading and subleading) photon pair, for a radion mass of $250 \mathrm{GeV}$. The prospects for restricting the mixed Higgs radion state parameter space at the LHC operating at 13 and $14 \mathrm{TeV}$ are shown in Fig. 6. We consider integrated luminosities of 50 and $150 \mathrm{fb}^{-1}$ for the $13 \mathrm{TeV}$ LHC and 300, 1000, and $3000 \mathrm{fb}^{-1}$ for the $14 \mathrm{TeV}$ LHC. The green and the green + blue regions indicate the values of the mixing parameter $\xi$ that can be observed with more than $3 \sigma$ significance level at 50 and $150 \mathrm{fb}^{-1}$ in the diphoton channel at $13 \mathrm{TeV}$ center of mass energy. The areas enclosed by red, cyan, and violet 

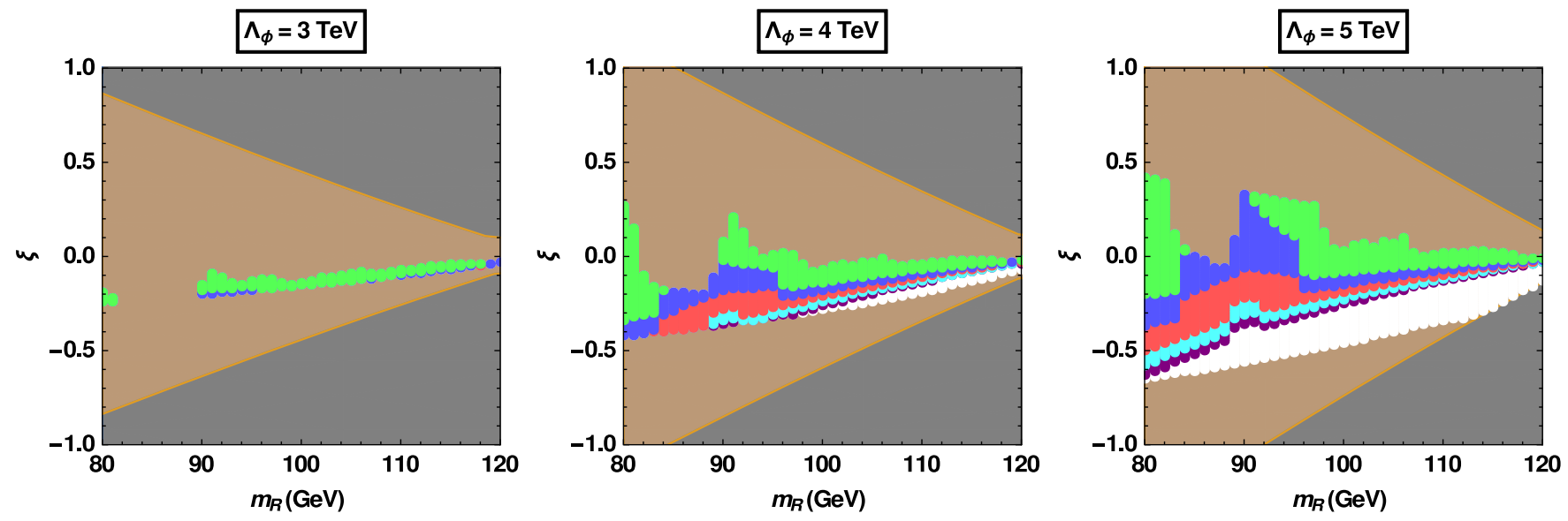

FIG. 6. Projected region 1 of the parameter space for $\Lambda_{\phi}=3,4,5 \mathrm{TeV}$ for a light mixed state. The brown region is the theoretically allowed region, while the dark gray region is theoretically disallowed. Green, blue, red, cyan, and violet colored regions represent regions which can be probed with 50 and $150 \mathrm{fb}^{-1}$, both for $13 \mathrm{TeV} \mathrm{LHC}$, and 300, 1000, and $3000 \mathrm{fb}^{-1}$ integrated luminosity for the $14 \mathrm{TeV}$ LHC, respectively. The interior white region (nonexistent for $\Lambda_{\phi}=3 \mathrm{TeV}$, small for $\Lambda_{\phi}=4 \mathrm{TeV}$, but larger for $\Lambda_{\phi}=5 \mathrm{TeV}$ ) represents the parameter region which cannot be probed.

are the regions that can be probed by the $14 \mathrm{TeV}$ LHC, with an integrated luminosity of 300,1000 , and $3000 \mathrm{fb}^{-1}$, respectively. We find that most of the $\xi$ region can be completely probed at $13 \mathrm{TeV}$ using the diphoton channel. With the increase of the center of mass energy from 13 to $14 \mathrm{TeV}$, we found that a higher mass of region 1 can be probed effectively. In Fig. 7, we show the regions of the $\xi$ parameter space that can be probed for a heavy radion. The color coding is similar to Fig. 6. We find that the diphoton channel can probe most of the parameter space of region 1 . The production rate of radion, decaying into two photons beyond $400 \mathrm{GeV}$, is too low and cannot be probed even at $3000 \mathrm{fb}^{-1}$. We next discuss the prospect of probing region 2 in the $Z Z$ decay channel of the radion.

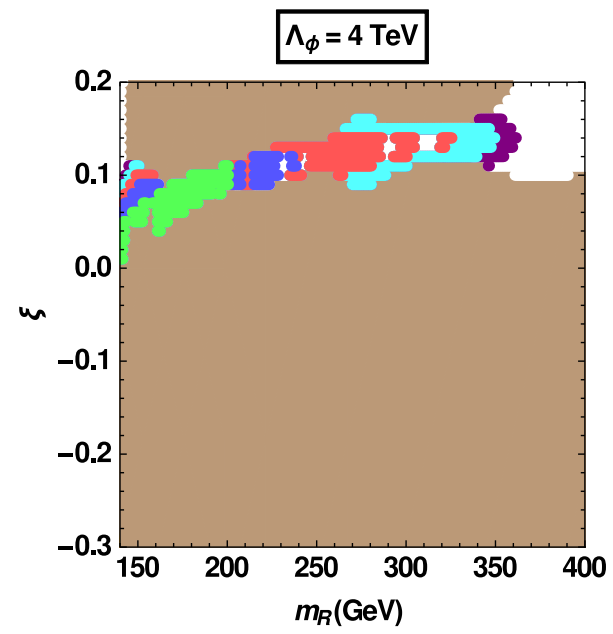

\section{Analysis in the $g g \rightarrow R \rightarrow Z Z, Z \rightarrow l \bar{l}$ decay channel}

When the mass of the radion is greater than $200 \mathrm{GeV}$, one can consider its decay to $Z Z$ and subsequently into four leptons. This channel also offers a clean reconstruction of the scalar mass. We considered gluon fusion of the scalar and its decay to $Z Z$. Although the production rate is not enough to probe the scenario at the $13 \mathrm{TeV} \mathrm{LHC}$, one can probe region 2, where the coupling of the heavy scalar to massive gauge bosons is large at the LHC $14 \mathrm{TeV}$ with higher integrated luminosity. To suppress combinatorial leptonic background, we consider a pair of electrons and a pair of muons as our signal, following the analysis given in Ref. [71]. The main irreducible background comes from the SM ZZ production. As before, we have generated the signal

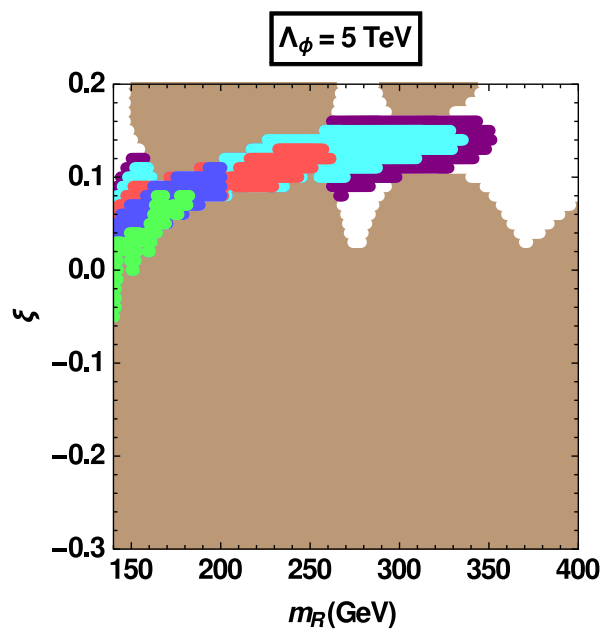

FIG. 7. Projection of region 1 parameter space at 13 and $14 \mathrm{TeV}$ for $\Lambda_{\phi}=4 \mathrm{TeV}$ (left panel) and for $\Lambda_{\phi}=5 \mathrm{TeV}$ (right panel), for a heavier mixed state. The brown region is the theoretically allowed region. The color coding is as in Fig. 6. 

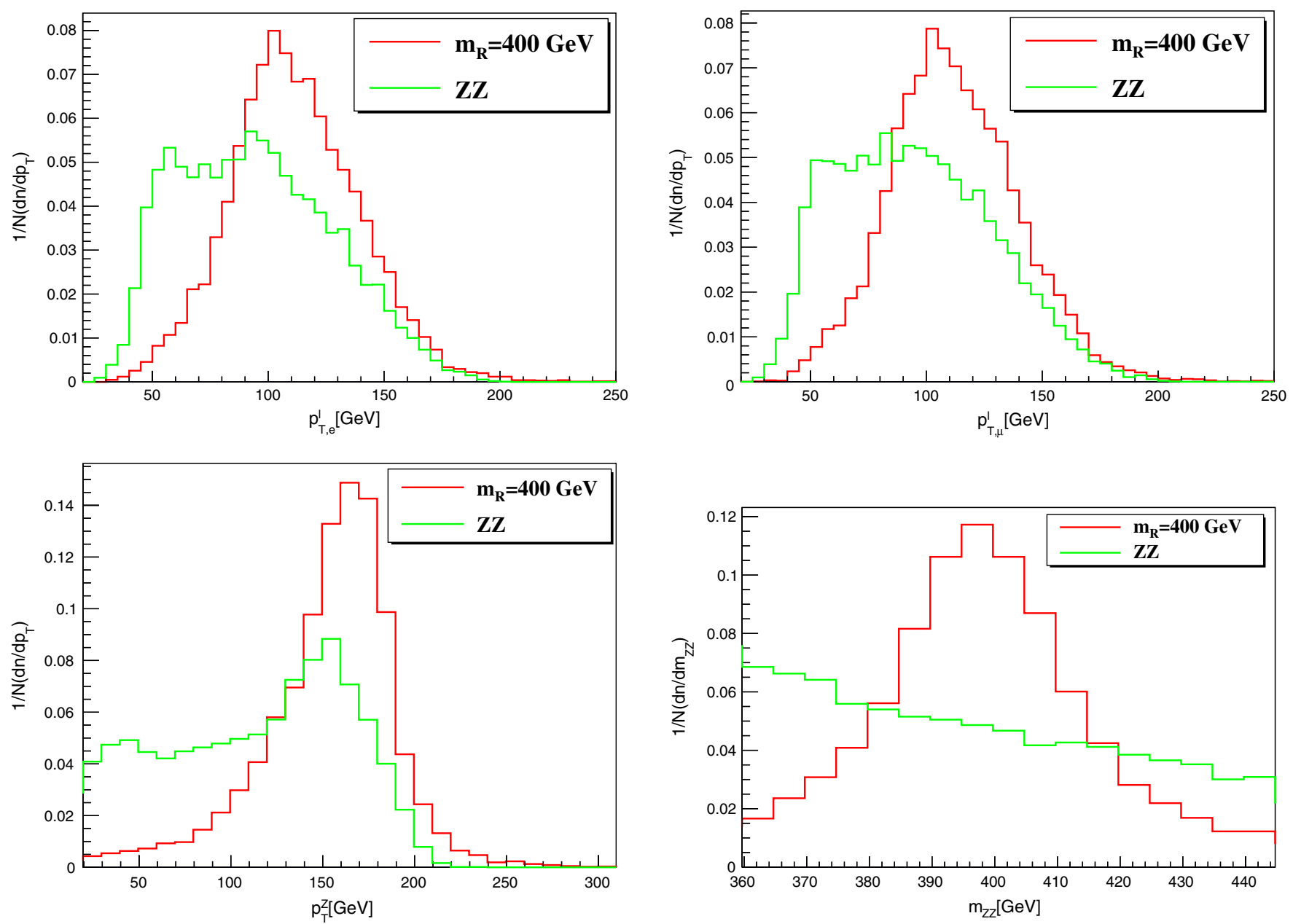

FIG. 8. The $p_{T}$ distribution of the leading electron (top-left panel), leading muon (top-right panel), of the reconstructed Z (bottom-left panel), and the invariant mass distribution of the $\mathrm{Z}$ boson (bottom-left panel) for a radion of mass of $400 \mathrm{GeV}$. The red line represents the signal, and the green line represents the background.

and the background events with showering and hadronization at the leading order in PYTHIA 8. We have used CTEQ611 as our PDF. The renormalization and factorization scales for both the signal and the background are kept at their default values. We selected events with no associated jets. The jet-veto cut helps us to get rid of the background coming from $t \bar{t} Z$. We consider two electrons having $p_{T}>17 \mathrm{GeV}$ and $\eta<2.5$ and two muons with $p_{T}>17 \mathrm{GeV}$ and $\eta<2.1$. The leptons are considered isolated if the scalar sum of the $p_{T}$ deposited within the cone of $\Delta R=0.3$ about the lepton is less than $10 \%$ of the $p_{T}$ of the lepton. We considered such pairs of isolated electron and muon with $p_{T}>25 \mathrm{GeV}$. We ensure that the pairs of same flavor leptons reconstruct the $Z$ mass. Events with transverse momentum of the same flavor dilepton system greater than $55 \mathrm{GeV}$ are selected. This selection cut helps us to control the irreducible background as shown in Fig. 8 (bottom left). These four leptons are further considered in our analysis.
For $m_{R}<300 \mathrm{GeV}$, we demand that the two reconstructed Z's have invariant mass lying within the window of $10 \mathrm{GeV}$ centered about the radion mass. We plot the normalized $p_{T}$ distribution of the leading electron and leading muon in Fig. 8. As the mass of the radion increases, the $Z$ 's are boosted, and hence leptons carry higher $p_{T}$. In order to increase the signal significance further, we consider events with a leading electron and muon having

$$
\begin{array}{ll}
p_{T}>50 \mathrm{GeV} & \text { for } 300 \mathrm{GeV}<m_{R}<400 \mathrm{GeV}, \\
p_{T}>80 \mathrm{GeV} & \text { for } 400 \mathrm{GeV}<m_{R}<500 \mathrm{GeV}
\end{array}
$$

and

$$
p_{T}>100 \mathrm{GeV} \text { for } 500 \mathrm{GeV}<m_{R}<800 \mathrm{GeV} \text {. }
$$

In this mass range, we have loosened the invariant mass cut by $20 \mathrm{GeV}$. Instead of applying a minimum cut on the 

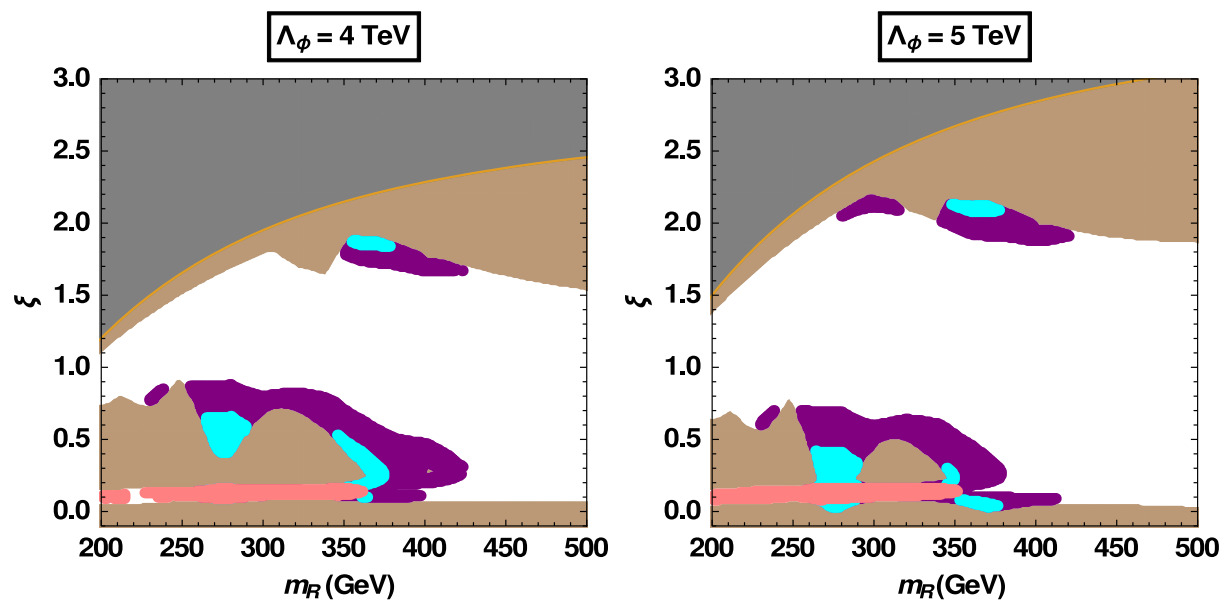

FIG. 9. Projection of region 1 and region 2 parameter space at $14 \mathrm{TeV}$ for $\Lambda_{\phi}=4 \mathrm{TeV}$ (left panel) and for $\Lambda_{\phi}=5 \mathrm{TeV}$ (right panel). The brown (gray) region is the theoretically allowed (disallowed) region; the pink region represents the area probed by diphoton channel at $3000 \mathrm{fb}^{-1}$. Cyan and violet colored regions represent regions which can be probed with 1000 and $3000 \mathrm{fb}^{-1}$ integrated luminosity, respectively, by $Z Z \rightarrow 4$ leptons. The interior white region represents the region which cannot be probed at the LHC $14 \mathrm{TeV}$.

transverse momentum of the leading lepton $\left(p_{T}^{1(\mathrm{e}, \mu}\right)$, one can apply a minimum cut on the transverse momentum of the reconstructed $Z$ boson, which has the same distinct feature as shown in Fig. 8. The reach of the $14 \mathrm{TeV}$ LHC to probe the allowed region of the Higgs-radion mixing using the $Z Z$ channel is shown in Fig. 9. We consider integrated luminosity of 300,1000 , and $3000 \mathrm{fb}^{-1}$ at $14 \mathrm{TeV}$. The area denoted by cyan represents the region that can be probed at $1000 \mathrm{fb}^{-1}$. The area denoted by cyan + purple represents the region that can be probed at the $3000 \mathrm{fb}^{-1}$. We find that using the $Z Z$ channel, one can probe the radion mass up to $450 \mathrm{GeV}$. The product of the coupling of the radion to gluon pair and radion to $Z$ pair is small in the parameter space allowed after the LHC $8 \mathrm{TeV}$, and hence the production rate of the radion via gluon fusion and its decay to $Z Z$ is relatively suppressed in this region. Thus, we need high luminosity to probe this area. In spite of such high luminosity, only the boundary of the region 2 has been probed effectively. One can also consider the production of the radion via vector boson fusion and its decay to a $Z$ boson. However, the cross section is suppressed for $\Lambda_{\phi}=4 \mathrm{TeV}$, and demanding that the $Z$ 's further decay to leptons decreases the production rate further. To probe this scenario more efficiently, we considered this region at the ILC in the next section.

\section{PROSPECTS FOR THE SEARCHES AT THE ILC}

Next, we perform a detailed study of the production cross section of the mixed radion at the ILC and its subsequent decay into the different allowed final states in region 2. As discussed before, the radion at the LHC could be analyzed mainly through the $\gamma \gamma$ final state. The other prominent decay modes, $g g$ and $b \bar{b}$, for
$m_{R}<180 \mathrm{GeV}$, will have a large background in the LHC and therefore cannot be tested thoroughly. The direct search for the radion in the ILC can be performed as long as it is kinematically accessible, and through it decay either into a dijet final state or $W W / Z Z$ final state, depending on its mass. Since the radion couples much like the Higgs, the production process will be analogous with the Higgs. It will be produced through the channel $e^{+} e^{-} \rightarrow Z R$ (associated production with $Z$, denoted as $Z R$ ) and $e^{+} e^{-} \rightarrow \nu_{e} \bar{\nu}_{e} R$ ( $W W$ fusion, denoted as $W W R$ ). We do not consider the $e^{+} e^{-} \rightarrow e^{+} e^{-} R$ (ZZ fusion) process here, as it has a very small cross section and will therefore require a high luminosity for any meaningful results. We show the total production cross section for $\sqrt{s}$ of 250,500 , and $1000 \mathrm{GeV}$, with the respective integrated luminosities of 250,500 , and $1000 \mathrm{fb}^{-1}$ in Fig. 10, for $\xi=0$ and $\Lambda_{\phi}=4 \mathrm{TeV}$, with unpolarized beams. It can be seen from Fig. 10 that $\sigma_{W W R}$ increases with $\sqrt{s}$, whereas $\sigma_{Z R}$, decreases. We consider the $Z R$ process, at $\sqrt{s}=250 \mathrm{GeV}$, as it is the dominant one. We first study the leptonic decay mode of $Z$, with $Z \rightarrow l \bar{l}$, where $l=e^{-}, \mu^{-}$. Since the leptonic branching ratio of $Z$ is small, we also considered the hadronic decay mode. The analysis at $\sqrt{s}=500$ and $1000 \mathrm{GeV}$ is through $W W R$, which is dominant at those energies.

\section{A. Analysis in the $e^{+} e^{-} \rightarrow Z R, Z \rightarrow \bar{l}, R \rightarrow b \bar{b}$ decay channel}

The $Z R$ production process considered here will consist of two leptons and two $b$ jets in the final state. At the ILC, due to a large cross section of the Bhabha scattering $e^{+} e^{-} \rightarrow e^{+} e^{-}$and the muon pair production $e^{+} e^{-} \rightarrow \mu^{+} \mu^{-}$, preselection cuts are applied on the leptons, in order to reduce the simulation time [72]. These cuts are as follows: 

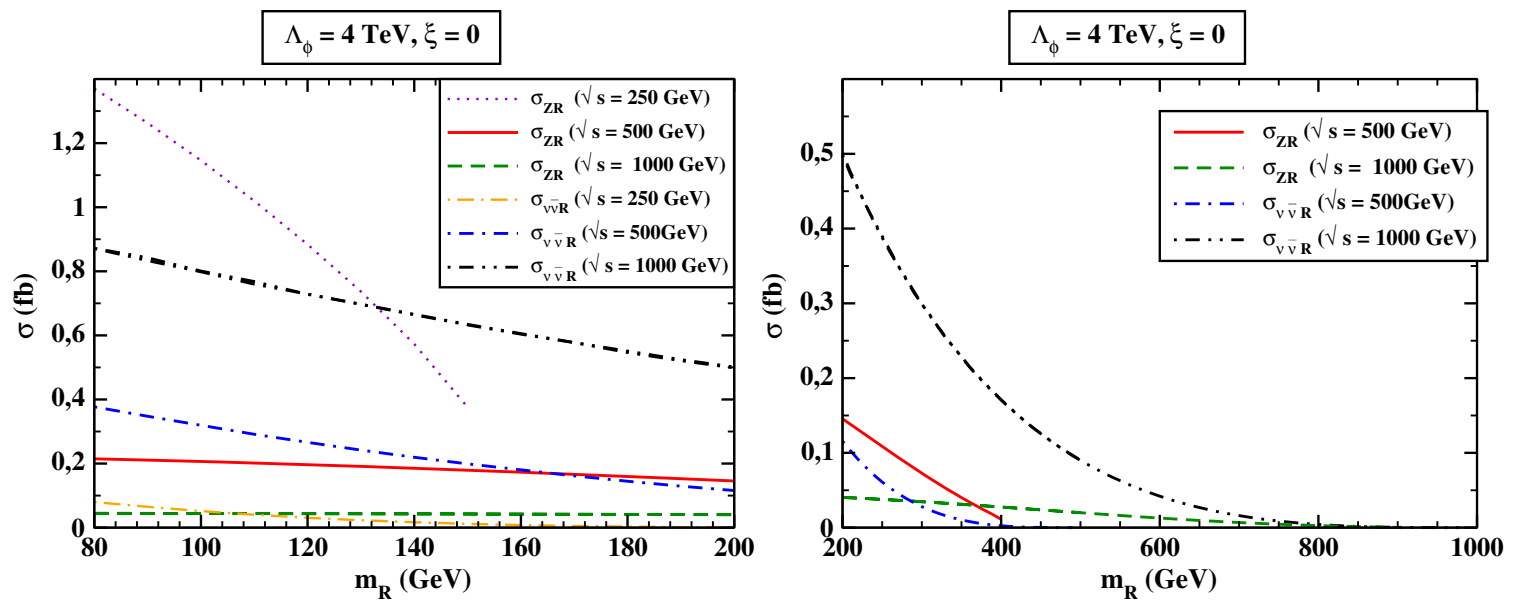

FIG. 10. The total production cross section of the unmixed radion at the ILC, as a function of the radion mass for $\Lambda_{\phi}=4$ TeV. Different contributions coming from $Z R$ and $W W R$ production at various $\sqrt{s}=250,500$, and $1000 \mathrm{GeV}$ are as indicated in legend on the right.

(a) $\left|\cos \theta_{l}\right|<0.95$

(b) $m_{\overline{l l}} \in(80,100) \mathrm{GeV}$

(c) $P_{\bar{l}}^{T}>20 \mathrm{GeV}$

(d) $m_{\text {recoil }} \in m_{R} \pm 15 \mathrm{GeV}$

(e) $0.2<\left|\phi_{l}-\phi_{\bar{l}}\right|<3$.

Here, $m_{\overline{l l}}$ is the invariant mass of the dilepton system, $P_{\bar{l}}^{T}$ is the transverse momentum calculated from the vectorial sum of the two leptons, and the recoil mass $m_{\text {recoil }}$ is expressed as

$m_{\text {recoil }}^{2}=s-2 \sqrt{s} E_{\bar{l} \bar{l}}+m_{\overline{l l}}^{2}, \quad\left(E_{\bar{l}}=E_{l}+E_{\bar{l}}\right)$,

where $\sqrt{s}$ is the c.m. energy and $E_{l}$ and $E_{\bar{l}}$ are the energies of the two leptons. The last cut is on the difference between the azimuthal angle of the two leptons. The signal is selected by identifying two well-measured leptons in the final state, which yield an invariant mass peak around the $Z$ boson mass. The recoil mass of the system should give the mass of the radion. Additionally, the signal event should consist of two $b$-tagged jets, with an invariant mass peak around $m_{R} \pm 5 \mathrm{GeV}$. The signal and the background events with the showering and the hadronization are generated in PYTHIA 8 [69]. Jet formation is done through Fastjet-3.2.0 [73] using the built-in $k_{t}$ algorithm for $e^{+} e^{-}$collisions, which is similar to the Durham algorithm. A jet is tagged as a $b$ jet if it has a $b$ parton within a cone of $\Delta R<0.4$ with the jet axis, where $\Delta R=\sqrt{(\Delta \eta)^{2}+(\Delta \phi)^{2}}$. A tagging efficiency of $80 \%$ [74] is also incorporated. The main backgrounds for the process under study are as follows:

(1) $e^{+} e^{-} \rightarrow \gamma \gamma, \quad \gamma \rightarrow \bar{l}, b \bar{b}$

(2) $e^{+} e^{-} \rightarrow \gamma Z, \quad Z, \gamma \rightarrow \bar{l}, b \bar{b}$

(3) $e^{+} e^{-} \rightarrow Z Z, \quad Z \rightarrow \bar{l}, b \bar{b}$

(4) $e^{+} e^{-} \rightarrow Z H, \quad Z \rightarrow l \bar{l}, H \rightarrow b \bar{b}$

We added the contributions of the backgrounds for $\gamma \gamma, \gamma Z$, and $Z Z$ and presented our result as a single background. The dominant background to this process comes from the $\mathrm{ZH}$ final state. In order to account for the detector effects, the momenta of the leptons and the jets are smeared using the following parametrization. The jet energies are smeared [75] with the different contributions being added in quadrature,

$$
\frac{\sigma\left(E_{\mathrm{jet}}\right)}{E_{\mathrm{jet}}}=\frac{0.4}{\sqrt{E_{\mathrm{jet}}}} \oplus 2.5 \%
$$

The momentum of the lepton is smeared as a function of the momentum and the angle $\cos \theta$ of the emitted leptons [76],

$\frac{\sigma\left(P_{l}\right)}{P_{l}^{2}}=\left(\begin{array}{cc}a_{1} \oplus \frac{b_{1}}{P_{l}}, & |\cos \theta|<0.78 \\ \left(a_{2} \oplus \frac{b_{2}}{P_{l}}\right)\left(\frac{1}{\sin (1-|\cos \theta|)}\right) & |\cos \theta|>0.78\end{array}\right)$

with

$\left(a_{1}, b_{1}\right)=2.08 \times 10^{-5}(1 / \mathrm{GeV}), \quad 8.86 \times 10^{-4}$,

$\left(a_{2}, b_{2}\right)=3.16 \times 10^{-6}(1 / \mathrm{GeV}), \quad 2.45 \times 10^{-4}$.

The region below $100 \mathrm{GeV}$ can be probed with a great precision at the LHC, and therefore we do not consider that region here. Moreover, at the ILC, the region below $100 \mathrm{GeV}$ will have a large background from the $Z$ resonance peak. Therefore, we performed our analysis, for $m_{R} \geq 100 \mathrm{GeV}$. The recoil mass distribution is shown in Fig. 11, for a radion mass of $110 \mathrm{GeV}$ and $\Lambda_{\phi}=5 \mathrm{TeV}$. The recoil mass should be peaked near $m_{R}$, but due to the initial state radiation and the bremsstrahlung effect, the distribution is spread out. In Fig. 12, we show the allowed parameter space that can be probed by the ILC at $\sqrt{s}=$ $250 \mathrm{GeV}$ through the $\mathrm{ZH}$ final state, with the $\mathrm{Z}$ decaying to two leptons. The theoretically allowed (disallowed) region, Eq. (24) is shown in brown (gray), whereas the region currently allowed by the LHC $8 \mathrm{TeV}$ results is shown in 


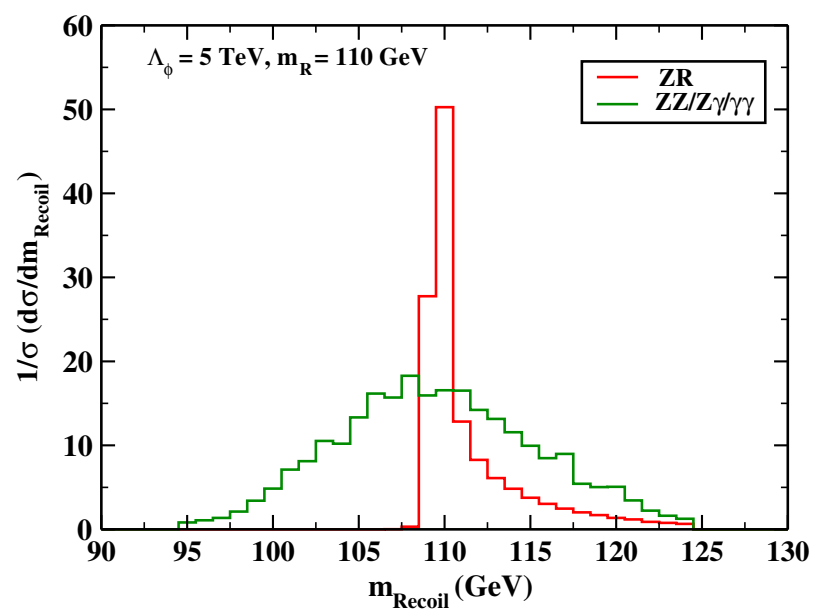

FIG. 11. The recoil mass distribution of the $e^{+} e^{-} \rightarrow Z R$ process at $\sqrt{s}=250 \mathrm{GeV}$, with unpolarized beams. The red line represents the signal, and the green line represents the background.

white. The regions probed with different luminosities are superimposed over each other and on the white region. The region in green denotes the values of the mixing parameter $\xi$ that can be observed with more than $3 \sigma$ significance level at $250 \mathrm{fb}^{-1}$, the regions in green and blue denote the probed regions at $500 \mathrm{fb}^{-1}$, and the red region along with green and blue can be probed with $1000 \mathrm{fb}^{-1}$. As the branching ratio of $Z$ to the leptonic final state is small, only a narrow region can be probed for $\Lambda_{\phi}$ of $4 \mathrm{TeV}$, with a high luminosity of $1000 \mathrm{fb}^{-1}$. But this channel can be used as a good probe for $\Lambda_{\phi}$ of $5 \mathrm{TeV}$, where it can probe the region which cannot be explored in LHC, shown in white in
Fig. 6. We next consider the hadronic decay mode of $Z$, with the aim to probe a larger region of the parameter space.

\section{B. Analysis in the $e^{+} e^{-} \rightarrow Z R, Z \rightarrow q \bar{q}, R \rightarrow b \bar{b}$ decay channel}

The analysis for this decay channel is similar to the leptonic decay mode of $Z$. The signal consists of four jets, and therefore events with isolated leptons are rejected. The jets are then constructed with the $k_{t}$ jet algorithm implemented in Fastjet, with the jet radius of 1.2 and the $p_{t}^{\min }=1.0 \mathrm{GeV}$, without restricting the number of the reconstructed jets $[77,78]$. Then, from all the jet pairs, a jet pair of mass consistent with $Z$ is selected. The recoil mass is calculated, and the cut on recoil mass is applied. The remaining jets are then checked for $b$ tagging as discussed before. The events with two $b$-tagged jets with an invariant mass peak around $m_{R} \pm 5 \mathrm{GeV}$ are then selected. The main backgrounds of the process are similar to the previous process with the $Z, \gamma$ decaying to quarks. There is an additional background from the $W W$ final state, with the $W$ 's decaying hadronically. We show in Fig. 13 the region in the $\xi-m_{R}$ plane that can be probed at $\sqrt{s}=250 \mathrm{GeV}$ for different values of integrated luminosities. The color coding is similar to the previous Fig. 12. The hadronic final state of $Z$ can probe a larger region of the parameter space compared to the leptonic decay mode. We do not consider this process for $\sqrt{s}=500 \mathrm{GeV}$ as the cross section falls with $\sqrt{s}$ and there the process $W W R$ yields better results.

\section{Analysis in the $e^{+} e^{-} \rightarrow \nu_{l} \bar{\nu}_{l} R, R \rightarrow b \bar{b}$ decay channel}

We next consider the measurement of the radion production cross section through $W W$ fusion at $\sqrt{s}=500$ and
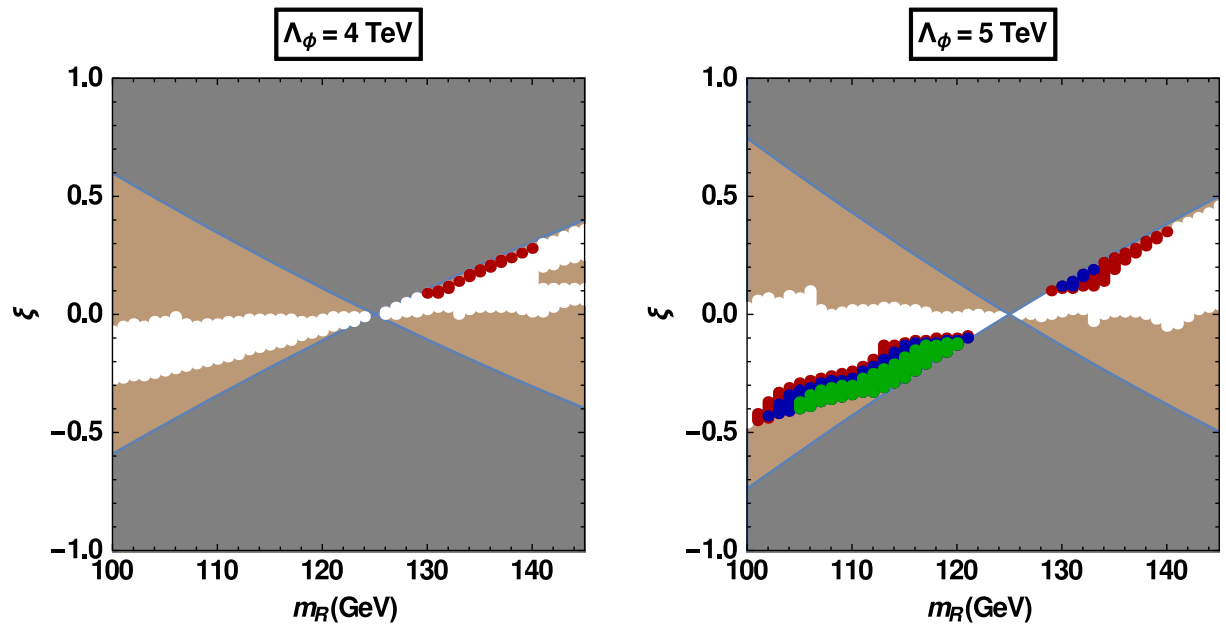

FIG. 12. Projected region 2 of the parameter space at $\sqrt{s}=250 \mathrm{GeV}$ for $\Lambda_{\phi}=4 \mathrm{TeV}$ (left panel) and for $\Lambda_{\phi}=5 \mathrm{TeV}$ (right panel), for a light mixed state through the $Z R, Z \rightarrow l \bar{l}, R \rightarrow b \bar{b}$ decay channels. The brown (dark gray) region is the theoretically allowed (disallowed) region. The green, green + blue, and green + blue + red colored regions represent areas which can be probed at 250, 500, and $1000 \mathrm{fb}^{-1}$ integrated luminosity, respectively. The interior white region is the one still allowed by the LHC $8 \mathrm{TeV}$ results which cannot be probed by the ILC through this final state. 

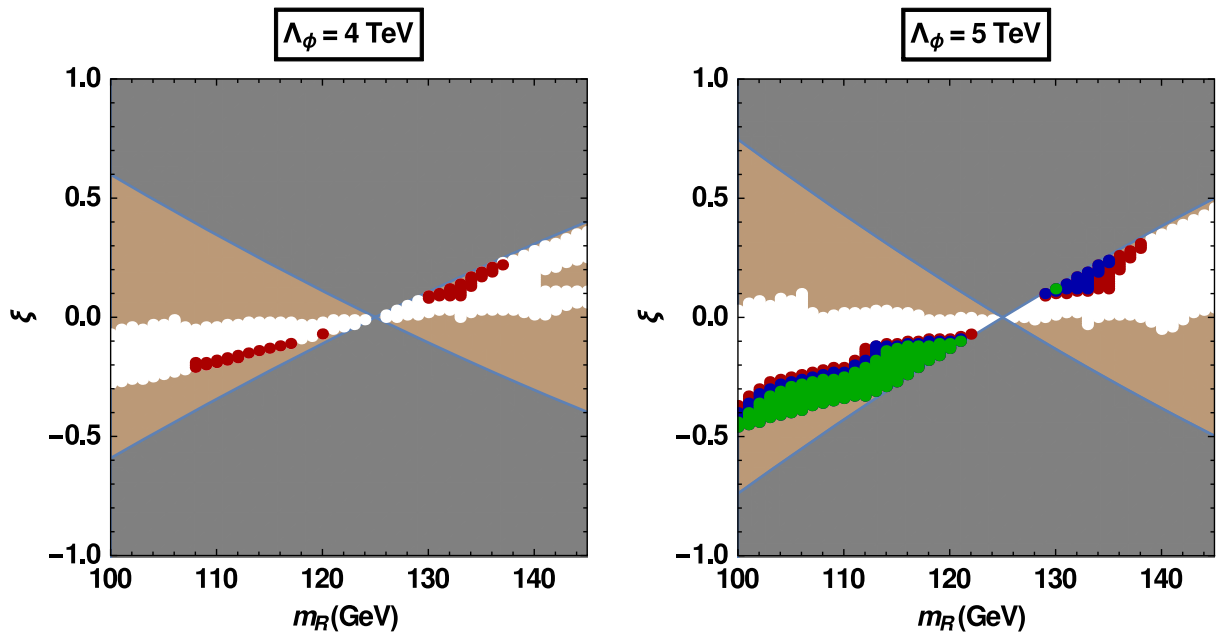

FIG. 13. Projected region 2 of parameter space at $\sqrt{s}=250 \mathrm{GeV}$ for $\Lambda_{\phi}=4 \mathrm{TeV}$ (left panel) and $\Lambda_{\phi}=5 \mathrm{TeV}$ (right panel), for a light mixed state through the $Z R, Z \rightarrow q \bar{q}, R \rightarrow b \bar{b}$ decay channel. The color coding is the same as in Fig. 12 .

$1000 \mathrm{GeV}$. We do not consider this process at $\sqrt{s}=250 \mathrm{GeV}$, as in the $\nu_{l} \bar{\nu}_{l} R$ final state, $Z R$, with $Z$ decaying to $\nu_{l} \bar{\nu}_{l}$ and $W W R$ cannot be treated separately. The $Z R$ cross section scales as $s^{-1}$, whereas the $W W R$ cross section increases logarithmically to large $\sqrt{s}$. Therefore, at low c.m. energies, $Z R$ will act as one of the most challenging backgrounds for $W W R$. The signal event consists of two energetic very forward $b$ jets along with missing 4-momentum. The main backgrounds in this search mode, apart from $Z R$, will be (1) two-jet production $(q \bar{q}),(2)$ semileptonically and hadronically decaying $Z / W$ pairs $\left(q \bar{q} q \bar{q}, q \bar{q} l \bar{l}, q \bar{q} \nu_{l} \nu_{\bar{l}}, q \bar{q} l \nu_{\bar{l}}\right)$, (3) single $Z / W$ - boson production process $\left(l \nu_{\bar{l}} W, e^{+} e^{-} Z\right)$ (4) $t \bar{t}$ production $(b \bar{b} W W)$, and (5) the SM Higgs production $\left(e^{+} e^{-} \rightarrow\right.$ $\left.\nu_{l} \bar{\nu}_{l} H, H \rightarrow b \bar{b}\right)$ through $W W$ fusion. Since the signal event consists of two $b$ jets along with missing energy, first the events with isolated leptons are rejected, and all the visible final particles are reconstructed into two jets. Jet formation is done with the same algorithm as discussed before for the $Z R$ process, with $Z$ decaying into two leptons. The lepton isolation cut subdues the background from semileptonically or leptonically decaying $W / Z$. The reconstructed jets are then tagged as $b$ jets, which significantly suppresses the hadronic background. The other selection cuts are:

(1) a cut on visible mass $\left(m_{\mathrm{vis}}\right)\left(m_{R} \pm 15 \mathrm{GeV}\right)$,

(2) a cut on visible energy $\left(5<E_{\mathrm{vis}}<m_{R}+150 \mathrm{GeV}\right)$,

(3) a cut on the missing mass $\left(m_{\text {missing }}>200 \mathrm{GeV}\right.$ for $\sqrt{s}=500 \mathrm{GeV}$ and $m_{\text {missing }}>400 \mathrm{GeV}$ for $\sqrt{s}=1000 \mathrm{GeV})$, and

(4) a cut on the reconstructed invariant radion mass $m_{R} \pm 10 \mathrm{GeV}$.

These cuts are sensitive to $\sqrt{s}$ as well as the mass of the radion. The background events from the hadronically decaying $W / Z$ have a lower missing mass, and therefore a higher cut on missing mass is applied. In Fig. 14, we show the visible mass, normalized visible energy, and invariant mass distribution for $m_{R}=150 \mathrm{GeV}$. From the left-hand figure, it can be seen that the cut on the visible mass results in reducing most of the background events, as the background is more spread out than the signal. The signal is not
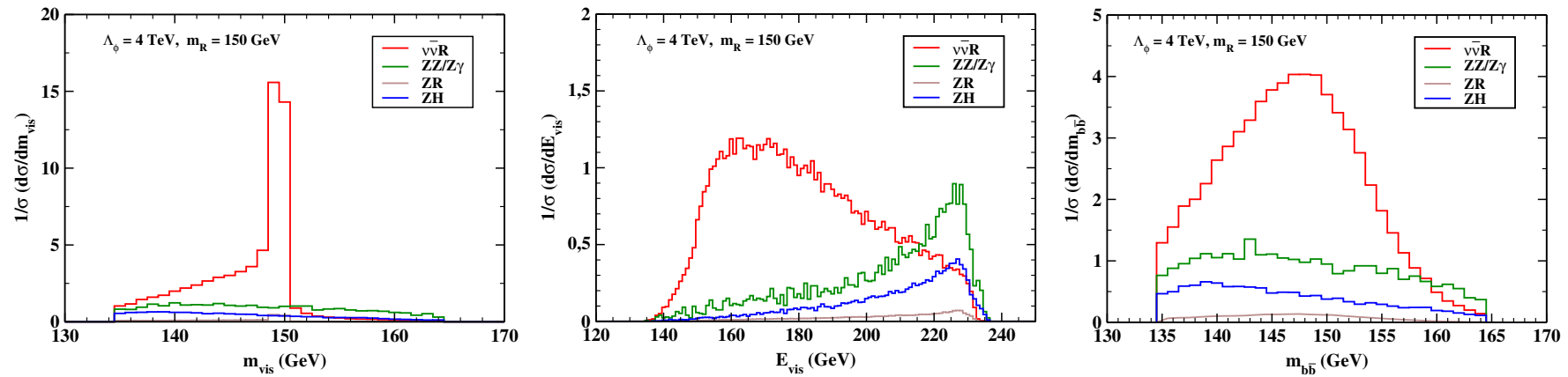

FIG. 14. The visible mass (left panel), visible energy (middle panel), and the invariant mass distribution (right panel) for a radion of mass $150 \mathrm{GeV}$ at $\sqrt{s}=500 \mathrm{GeV}$ and a luminosity of $500 \mathrm{fb}^{-1}$. The red line represents the signal, whereas the green, blue, and brown lines represent the background. 

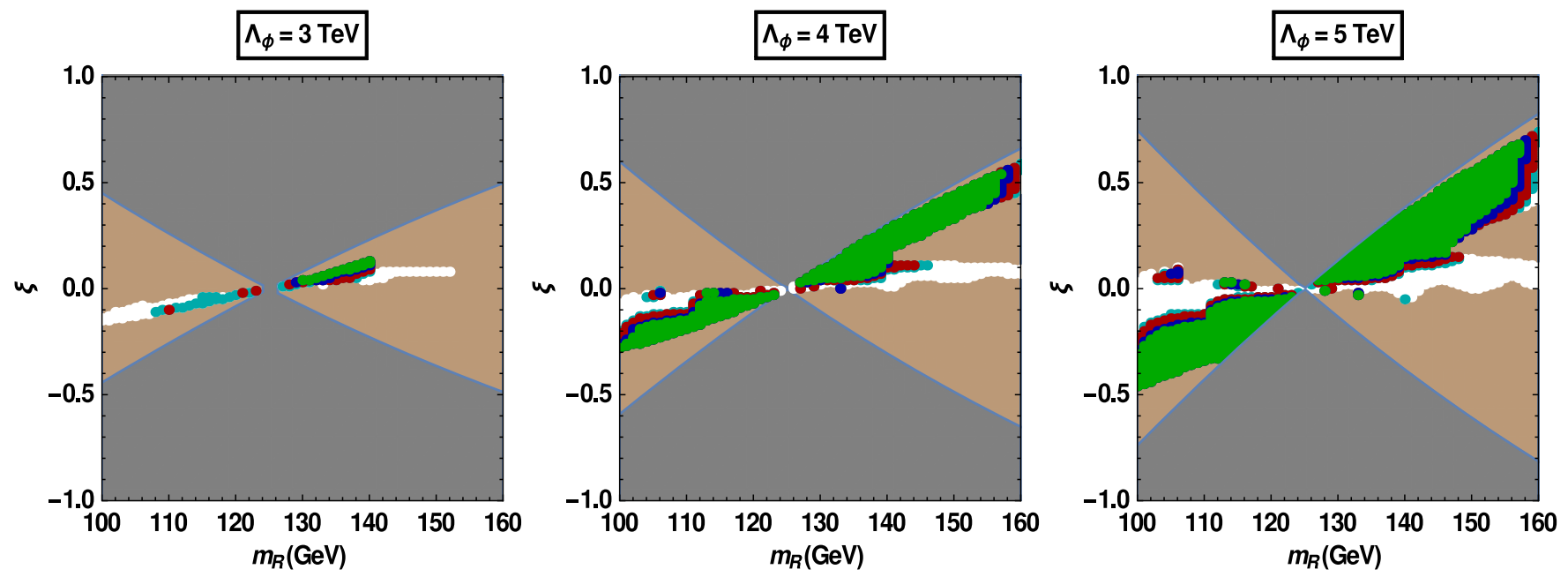

FIG. 15. Projected region 2 of the parameter space at $\sqrt{s}=500$ and $1000 \mathrm{GeV}$ for $\Lambda_{\phi}=3 \mathrm{TeV}$ (left panel), $\Lambda_{\phi}=4 \mathrm{TeV}$ (middle panel), and $\Lambda_{\phi}=5 \mathrm{TeV}$ (right panel) for a light mixed state. The green, green + blue, green + blue + red, and green + blue + red + cyan colored regions represent parameter space regions which can be probed at $\sqrt{s}=500, \mathcal{L}=500 \mathrm{fb}^{-1}, \sqrt{s}=500 \mathrm{GeV}$, $\mathcal{L}=1000 \mathrm{fb}^{-1}, \sqrt{s}=1000 \mathrm{GeV}, \mathcal{L}=500 \mathrm{fb}^{-1}$, and $\sqrt{s}=1000, \mathcal{L}=1000 \mathrm{fb}^{-1}$, respectively. The interior white region is the one still allowed by the LHC $8 \mathrm{TeV}$ results but which cannot be probed by the ILC through this final state.

peaked at $m_{R}$ but is a bit spread in the lower end, due to the loss of the beam's energy from the bremsstrahlung and the initial state radiation effects. The same effect can be seen in the visible energy distribution (middle panel) of the signal. An upper cut on $E_{\mathrm{vis}}$ reduces most of the background as the background peaks toward higher $E_{\text {vis }}$. Finally, on the right-hand panel of Fig. 14, we show the invariant mass reconstruction from the two $b$-tagged jets for both the signal and the background. We show in Fig. 15 the projected parameter space in the $\xi-m_{R}$ plane, through $W W$ fusion at $\sqrt{s}$ of 500 and $1000 \mathrm{GeV}$. The region in green is the region which can be probed at $\sqrt{s}=$ $500 \mathrm{GeV}$ with an integrated luminosity of $500 \mathrm{fb}^{-1}$, and the blue and green region is the one probed with $\sqrt{s}=$ $500 \mathrm{GeV}$ and $1000 \mathrm{fb}^{-1}$. The red, blue, and green region is the one which can be probed with $\sqrt{s}=1000 \mathrm{GeV}$ and an integrated luminosity of $500 \mathrm{fb}^{-1}$. Finally, the colored region including cyan, red, blue, and green is for $\sqrt{s}=$ $1000 \mathrm{GeV}$ and $1000 \mathrm{fb}^{-1}$. The parameter space in the $\xi-m_{R}$ plane for $\Lambda_{\phi}=3 \mathrm{TeV}$, which cannot be probed by the $Z R$ process, can be probed by $W W$ fusion. The $W W$ fusion process can test most of the $\xi-m_{R}$ parameter space for $\Lambda_{\phi}=3,4$, and $5 \mathrm{TeV}$. This is mainly because $W W R$ has a larger cross section compared to $Z R$ and, second, because $Z R$ is further suppressed through the selection of the $Z$ decay channel. The decay channel of the final state radion $R \rightarrow b \bar{b}$ can only probe the $m_{R}<160 \mathrm{GeV}$ region. The $W W, Z Z$ decay channels open up for higher values of $m_{R}$ and have a larger branching ratios compared to $b \bar{b}$. We therefore next consider the $W W$ decay channel, so as to probe the sensitivity of the ILC for higher radion mass.

\section{Analysis in the $e^{+} e^{-} \rightarrow \nu_{l} \bar{\nu}_{l} R, R \rightarrow W^{+} W^{-}$, $W \rightarrow q \bar{q}$ decay channel}

We finally analyze the $W W$ fusion process, with $R$ decaying to $2 W$ 's and the $W$ 's decaying hadronically. The final state in this decay mode consists of two missing neutrinos and four jets, none of which is a $b$ jet. The events with isolated leptons are first rejected. Then, the remaining particles are clustered into four jets with the jets clustering algorithms. The main background processes are similar to the $W W$ fusion $(R \rightarrow b \bar{b})$ analysis, dominated by $W^{+} W^{-}$and $\nu \bar{\nu} Z$. The jets for the signal are originating from $R$ which decays to $2 W$ 's, and therefore we demand that the total number of particles passing for the jet clustering algorithm be greater than 30 [79]. This reduces the background processes with the jets constructed from a single $W / Z$. The events with total $p_{T}<20 \mathrm{GeV}$ are rejected, and the cuts are applied on the visible mass, visible energy, and missing mass, similar to what we discussed in Sec. VIC. We do not show the $m_{\mathrm{vis}}, E_{\mathrm{vis}}$ distributions, as they will have the same pattern as Fig. 14. The four reconstructed jets are then paired to give two onshell $\mathrm{W}$ bosons, with $m_{W} \pm 11 \mathrm{GeV}$. The reconstructed radion invariant mass from the on-shell $W$ 's is required to be in the mass range $m_{R} \pm 10 \mathrm{GeV}$. The $b$ jet veto is used to reduce the background from $H / R \rightarrow b \bar{b}, t \bar{t}$ final states or the $t \bar{t}$ pair production. It is difficult to study this mode in the LHC as it yields a hadronic final state. The leptonically decaying $W$ 's can be studied in the LHC, but as discussed before, this will prove difficult with radion mass reconstruction due to missing neutrinos. We therefore study the all hadronic modes at the ILC and show, in Fig. 16, the parameter space that can be probed at the ILC, at $\sqrt{s}=500$ and $1000 \mathrm{GeV}$. The color coding is the same as Fig. 15. There is a narrow white region, 

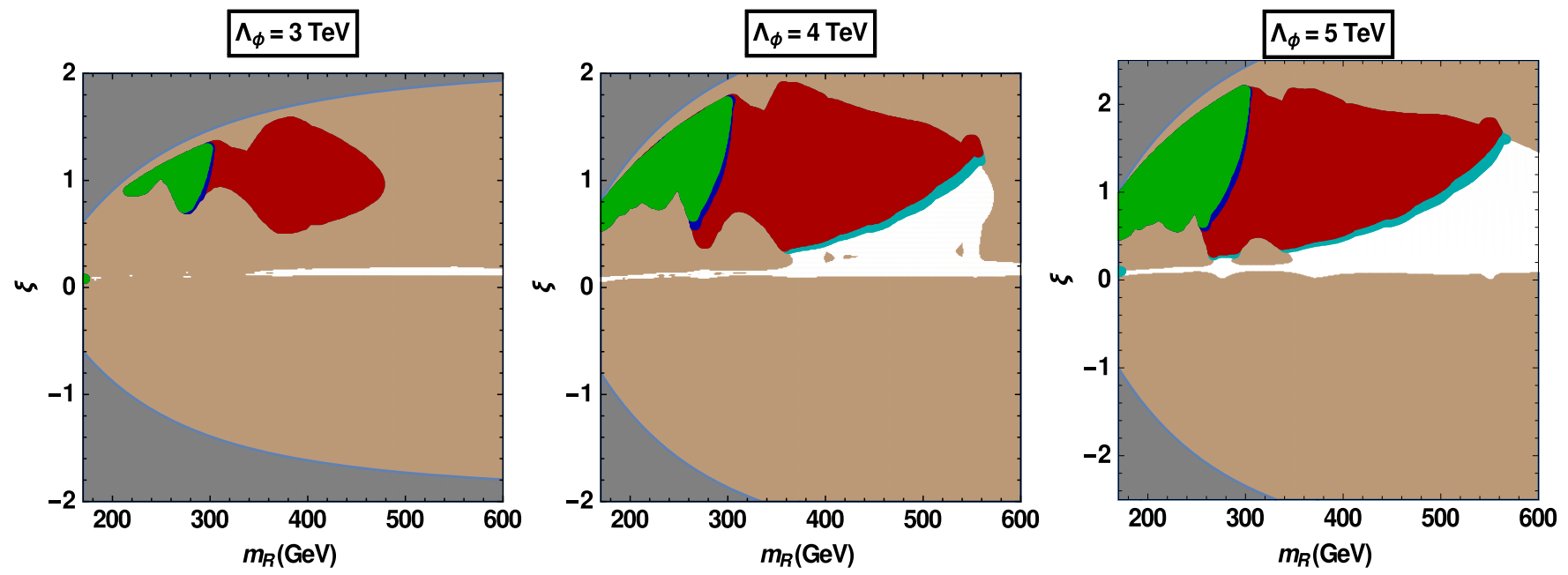

FIG. 16. Projected region 2 of the parameter space at $\sqrt{s}=500$ and $1000 \mathrm{GeV}$ for $\Lambda_{\phi}=3 \mathrm{TeV}$ (left panel), $\Lambda_{\phi}=4 \mathrm{TeV}$ (middle panel), and $\Lambda_{\phi}=5 \mathrm{TeV}$ (right panel), for a light mixed state. Green, blue, red, and cyan colored regions represent regions ruled out at $\left(\sqrt{s}=500, \mathcal{L}=500 \mathrm{fb}^{-1}\right),\left(\sqrt{s}=500, \mathcal{L}=1000 \mathrm{fb}^{-1}\right),\left(\sqrt{s}=1000, \mathcal{L}=500 \mathrm{fb}^{-1}\right)$, and $\left(\sqrt{s}=1000, \mathcal{L}=1000 \mathrm{fb}^{-1}\right)$, respectively. The interior white region is the one still allowed by the LHC $8 \mathrm{TeV}$ results, but cannot be probed by the ILC, through this final state.

near $\xi=0$, which cannot be probed at the ILC; this corresponds to region 1 , where the coupling of the radion with the SM fermions and the massive gauge bosons vanishes, and only the coupling with massless gauge bosons exists. A through study of the hadronically decaying $W W$ final state in the ILC will be able to constrain most of region 2 , for $170 \mathrm{GeV}<m_{R}<540 \mathrm{GeV}$. In the next section, we discuss how the LHC and the ILC analysis will complement each other in constraining the entire $\xi-m_{R}$ parameter space.

\section{DISCUSSIONS AND CONCLUSIONS}

In what follows, we summarize the results and indicate the conclusions to be drawn from our analysis. We have studied the phenomenology of the radionlike mixed state in the custodial RS model, where all the SM particles except the Higgs boson live in the bulk. We discussed the $\xi-m_{R}$ parameter space in the context of recent LHC results from the heavy Higgs, Higgs signal strength, and $750 \mathrm{GeV}$ resonance. There are still allowed regions in the $\xi-m_{R}$ parameter space, which can be thoroughly examined in the LHC and the planned ILC.

The existing parameter space depending on $\Lambda_{\phi}$ is divided in two regions for our analysis. Region 1, where the radion couples maximally to the massless gauge bosons, can be studied thoroughly in the 13 and $14 \mathrm{TeV}$ LHC, through the $\gamma \gamma$ final state. We discussed the prospect of direct searches for the radion at the LHC through the $\gamma \gamma$ decay mode and have shown the regions in the $\xi-m_{R}$ plane which can be probed this way. We find that with this decay channel the $14 \mathrm{TeV}$ LHC with an integrated luminosity of $1000 \mathrm{fb}^{-1}$ can probe region 1 of a radion in the mass range $80<m_{R}<350 \mathrm{GeV}$ with more than $3 \sigma$. There are also regions in the $\xi-m_{R}$ parameter space, for $m_{R} \leq 200 \mathrm{GeV}$, which the $13 \mathrm{TeV} \mathrm{LHC}$ can probe with an integrated luminosity of $50 \mathrm{fb}^{-1}$ only. Region 2, with the radion coupling maximally to the massive gauge bosons and $b$ quarks, can be studied in both the LHC and the ILC. The massive gauge bosons from the radion decay further decay into quarks or leptons. Though the all hadronic final state has a large LHC background, it is possible to discover a heavy radion through the leptonic decay modes of the gauge bosons in a high luminosity LHC. We therefore considered the $Z Z$ decay mode of the radion, with the $Z$ decaying leptonically, for the $14 \mathrm{TeV}$ LHC. We found that this channel extends the discovery reach of the LHC in the $\xi-m_{R}$ parameter space beyond what is tested through the diphoton mode, till $m_{R}$ of $450 \mathrm{GeV}$.

As seen in Figs. 6 and 9, for $\Lambda_{\phi}=4,5 \mathrm{TeV}$, there are regions in the parameter space where the LHC cannot discover the radion or cannot constrain the $\xi-m_{R}$ parameter space. This white region is region 2 where the radion decays dominantly to $b\left(m_{R}<160 \mathrm{GeV}\right)$ or $W W / Z Z$ $\left(m_{R}>160 \mathrm{GeV}\right)$. This region can be thoroughly investigated in the ILC, through the $Z R$ and the $W W R$ production mode, shown in Figs. 13, 15, and 16. In order to highlight the complementarity of the ILC and the LHC, we investigate some benchmark points for $m_{R}$ and show the range of $\xi$ ( $\xi_{\text {probed }}$ ) that can be explored by both the machines. We again note that for $m_{R}<100 \mathrm{GeV}$ the entire allowed parameter space of $\xi$ can be explored in the $\mathrm{LHC}$ for $\Lambda_{\phi}=3$ and $4 \mathrm{TeV}$. In Table I, we list the range of $\xi$ that can be probed at $3 \sigma$ and $5 \sigma$ for selected values of $m_{R}$ and for $\Lambda_{\phi}=4 \mathrm{TeV}$. We also list as $\xi_{\exp }$ the domain of $\xi$, which survives both the theoretical and the experimental constraints from the $8 \mathrm{TeV}$ LHC. The production and the decay modes of the radion considered for the analysis are also listed in the table. We presented the results for the LHC for a luminosity of $150 \mathrm{fb}^{-1}$ 
TABLE I. The range of $\xi\left(\xi_{\text {probed }}\right.$ ) that can be tested at the ILC and the LHC, in conjunction with considered values of $m_{R}$, for $\Lambda_{\phi}=4 \mathrm{TeV}$. The allowed region of $\xi$ from the theory and the $8 \mathrm{TeV} \mathrm{LHC}$ results is denoted by $\xi_{\text {exp }}$. The mass and the $\xi$ region which cannot be probed through the $\gamma \gamma, Z Z$ final state in the LHC and the $b \bar{b}, W^{+} W^{-}$final state in the ILC is denoted by $(\times \times)$.

\begin{tabular}{|c|c|c|c|c|}
\hline$\left(m_{R}, \xi_{\exp }\right)$ & Production mode & Decay mode & $\xi_{\text {probed }}^{3 \sigma}$ & $\xi_{\text {probed }}^{5 \sigma}$ \\
\hline $110 \mathrm{GeV},(-0.19--0.04)$ & $\begin{array}{c}g g \rightarrow R(\mathrm{LHC} 13) \\
g g \rightarrow R\left(\mathrm{LHC}_{14}\right) \\
Z R, Z \rightarrow q \bar{q}\left(\mathrm{ILC}_{1000}^{250}\right) \\
W W R\left(\mathrm{ILC}_{500}^{1000}\right)\end{array}$ & $\begin{array}{l}R \rightarrow \gamma \gamma \\
R \rightarrow \gamma \gamma \\
R \rightarrow b \bar{b} \\
R \rightarrow b \bar{b}\end{array}$ & $\begin{array}{l}-0.09--0.04 \\
-0.14--0.04 \\
-0.19--0.17 \\
-0.19--0.11\end{array}$ & $\begin{array}{c}-0.07--0.04 \\
-0.13--0.04 \\
\times \times \\
-0.19--0.12\end{array}$ \\
\hline $140 \mathrm{GeV},(0.01-0.28)$ & $\begin{array}{c}g g \rightarrow R(\mathrm{LHC} 13) \\
g g \rightarrow R\left(\mathrm{LHC}^{4}\right) \\
Z R, Z \rightarrow q \bar{q}\left(\mathrm{ILC}_{1000}^{250}\right) \\
W W R\left(\mathrm{ILC}_{500}^{500}\right) \\
W W R\left(\mathrm{ILC}_{1000}^{1000}\right)\end{array}$ & $\begin{array}{l}R \rightarrow \gamma \gamma \\
R \rightarrow \gamma \gamma \\
R \rightarrow b \bar{b} \\
R \rightarrow b \bar{b} \\
R \rightarrow b \bar{b}\end{array}$ & $\begin{array}{c}0.01-0.06 \\
0.01-0.09 \\
0.28 \\
0.13-0.28 \\
0.09-0.28\end{array}$ & $\begin{array}{l}0.01-0.04 \\
0.01-0.08 \\
\quad \times \times \\
0.15-0.28 \\
0.1-0.28\end{array}$ \\
\hline $200 \mathrm{GeV},(0.09-0.11,0.78-1.05)$ & $\begin{array}{c}g g \rightarrow R(\mathrm{LHC} 13) \\
g g \rightarrow R(\mathrm{LHC} 14) \\
g g \rightarrow R\left(\mathrm{LHC}^{4}\right) \\
W W R\left(\mathrm{ILC}_{500}^{500}\right) \\
W W R\left(\mathrm{ILC}_{500}^{1000}\right)\end{array}$ & $\begin{array}{l}R \rightarrow \gamma \gamma \\
R \rightarrow \gamma \gamma \\
R \rightarrow Z Z \\
R \rightarrow W^{+} W^{-} \\
R \rightarrow W^{+} W^{-}\end{array}$ & $\begin{array}{l}0.09-0.11 \\
0.09-0.11 \\
\quad \times \times \\
0.78-1.05 \\
0.78-1.05\end{array}$ & $\begin{array}{l}0.09-0.11 \\
0.09-0.11 \\
\quad \times \times \\
0.78-1.05 \\
0.78-1.05\end{array}$ \\
\hline $280 \mathrm{GeV},(0.09-0.16,0.44-1.64)$ & $\begin{array}{c}g g \rightarrow R(\mathrm{LHC} 13) \\
g g \rightarrow R(\mathrm{LHC} 14) \\
g g \rightarrow R(\mathrm{LHC} 14) \\
W W R\left(\mathrm{ILC}_{1000}^{500}\right) \\
W W R\left(\mathrm{ILC}_{500}^{1000}\right)\end{array}$ & $\begin{array}{l}R \rightarrow \gamma \gamma \\
R \rightarrow \gamma \gamma \\
R \rightarrow Z Z \\
R \rightarrow W^{+} W^{-} \\
R \rightarrow W^{+} W^{-}\end{array}$ & $\begin{array}{c}\times \times \\
0.09-0.16 \\
0.09-0.16,0.44-0.88 \\
1.67-1.74 \\
0.73-1.74\end{array}$ & $\begin{array}{c}\times \times \\
0.09-0.16, \\
0.09-0.16,0.44-0.67 \\
\times \times \\
0.73-1.74\end{array}$ \\
\hline $400 \mathrm{GeV},(0.11-1.72)$ & $\begin{array}{c}g g \rightarrow R(\mathrm{LHC} 13) \\
g g \rightarrow R(\mathrm{LHC} 14) \\
g g \rightarrow R(\mathrm{LHC} 14) \\
W W R\left(\mathrm{ILC}_{1000}^{500}\right) \\
W W R\left(\mathrm{ILC}_{1000}^{1000}\right)\end{array}$ & $\begin{array}{l}R \rightarrow \gamma \gamma \\
R \rightarrow \gamma \gamma \\
R \rightarrow Z Z \\
R \rightarrow W^{+} W^{-} \\
R \rightarrow W^{+} W^{-}\end{array}$ & $\begin{array}{c}\times \times \\
\times \times \\
0.2-0.47,1.67-1.72 \\
\times \times \\
0.43-1.72\end{array}$ & $\begin{array}{c}\times \times \\
\times \times \\
\times \times \\
\times \times \\
0.52-1.72\end{array}$ \\
\hline $500 \mathrm{GeV},(0.11-1.49)$ & $\begin{array}{c}g g \rightarrow R(\mathrm{LHC} 13) \\
g g \rightarrow R(\mathrm{LHC} 14) \\
g g \rightarrow R(\mathrm{LHC} 14) \\
W W R\left(\mathrm{ILC}_{500}^{1000}\right) \\
W W R\left(\mathrm{ILC}_{1000}^{1000}\right)\end{array}$ & $\begin{array}{l}R \rightarrow \gamma \gamma \\
R \rightarrow \gamma \gamma \\
R \rightarrow Z Z \\
R \rightarrow W^{+} W^{-} \\
R \rightarrow W^{+} W^{-}\end{array}$ & $\begin{array}{c}x \times \\
\times \times \\
\times \times \\
0.89-1.49 \\
0.79-1.49 \\
\end{array}$ & $\begin{array}{c}\times \times \\
\times \times \\
\times \times \\
1.07-1.49 \\
0.95-1.49\end{array}$ \\
\hline
\end{tabular}

for $\sqrt{s}=13 \mathrm{TeV}$ (LHC13) and $3000 \mathrm{fb}^{-1}$ for $\sqrt{s}=$ $14 \mathrm{TeV}$ (LHC14). In case of the ILC, the smallest possible combination of $\sqrt{s}$ and $\mathcal{L}$, which can probe the maximum $\xi-m_{R}$ parameter space, is listed ( $\operatorname{ILC}_{\mathcal{L}}^{\sqrt{s}}$ ).

We briefly discuss the results for $\Lambda_{\phi}=3 \mathrm{TeV}$ before discussing the results for $4 \mathrm{TeV}$. The allowed range of $\xi-m_{R}$ for $\Lambda_{\phi}=3 \mathrm{TeV}$ is tightly constrained by the LHC results. From Fig. 6, it can be seen that a radion of mass up to $120 \mathrm{GeV}$ can be fully explored by the LHC. The ILC will be at a disadvantage for this low mass range because of a large background from the $Z$ resonance and the SM Higgs. The complementarity of the ILC and the LHC is therefore visible for $m_{R}>140 \mathrm{GeV}$. The experimentally allowed range of $\xi$ for $m_{R}=140 \mathrm{GeV}$ is (0.04-0.13), and we find that the $14 \mathrm{TeV}$ LHC with $3000 \mathrm{fb}^{-1}$ can probe at $3 \sigma$ the lower $\xi$ range [(0.04-0.09) region 1$]$, through the diphoton decay channel. The 500(1000) GeV ILC with integrated luminosity of $500(1000) \mathrm{fb}^{-1}$ probes the $\xi$ range $[0.11$ (0.08)-0.13]. This clearly shows how the two colliders will complement each other in probing the $\xi-m_{R}$ parameter space. The radion of mass greater than $250 \mathrm{GeV}$ cannot be probed by the diphoton channel of the LHC but can be probed through the $Z Z$ final state, with the $Z$ decaying leptonically. The ILC running at a higher c.m. energy and higher luminosity can also probe this high mass region.

We next present the results for $\Lambda_{\phi}=4 \mathrm{TeV}$, in Table I, where the complementarity of the ILC and the LHC is more pronounced for $m_{R}<300 \mathrm{GeV}$. Let us consider $m_{R}=140 \mathrm{GeV}$, for which the allowed region is $\xi_{\exp }=(0.01-0.28)$. It can be seen from the table that the $14 \mathrm{TeV}\left(3000 \mathrm{fb}^{-1}\right)$ LHC, through the diphoton channel, and the ILC operating at $\sqrt{s}=1000 \mathrm{GeV}$ can fully explore the parameter space with $1000 \mathrm{fb}^{-1}$. The same argument holds for the radion mass in the range of 200 to $400 \mathrm{GeV}$. The available parameter space for the radion with 
mass less than $400 \mathrm{GeV}$ consists of two distinct regions, one near $\xi=0$ and another for $\xi>0.5$. The narrow band near $\xi=0$ is fully explored by the LHC through the diphoton channel. The $Z Z$ decay channel can help the LHC to probe the region with $\xi>0.5$, but it cannot fully explore the region. However, the $1000 \mathrm{GeV}$ ILC, through the $W W R$ production mode, can fully probe the $\xi>0.5$ parameter space. The two distinct regions of $\xi$ merge for $m_{R} \geq 400 \mathrm{GeV}$, and this mass range cannot be tested at the LHC through the diphoton channel. The $W W R$ channel in the ILC can only probe the upper region of $\xi$. The LHC analysis of the radion in the $Z Z$ decay channel helps in probing the region for this mass, which cannot be explored in the ILC. However, the $Z Z$ decay channel can only probe for a radion of mass less than $450 \mathrm{GeV}$, after which the production cross section is too small to be observed in the LHC. The $1000 \mathrm{GeV}$ ILC can also probe, through the $W W R$ channel, regions of $\xi$ for heavier radions as long as the radion mass is kinematically accessible.

In conclusion, we showed that, using the coherent analysis at the ILC and the LHC, the mixed Higgs-radion state can be revealed, its mass can be verified, and the mixing sector can be fully explored.

\section{ACKNOWLEDGMENTS}

M.F. thanks NSERC for partial financial support under Grant No. SAP105354. K. H. acknowledges the H2020-MSCA-RICE-2014 Grant No. 645722 (NonMinimalHiggs). U. M. and M. P. would like to thank Abhishek M. Iyer and Tousik Samui for discussions. U. M. would like to thank the Department of Theoretical Physics, TIFR, for the use of its computational resources. U.M. would also like to thank the hospitality of Concordia University, Montreal, and Helsinki Institute of Physics, Helsinki, where this work was initiated. M. P. would like to thank the Department of Theoretical Particle Physics, LMU, Munich, for hospitality during the initial stage of this work. The work of M. P. is supported by the Croatian Science Foundation (HrZZ) project "Physics of Standard Model and Beyond," HrZZ 5169 as well as by the H2020 Twinning project, "RBI-T-WINNING."
[1] N. Arkani-Hamed, S. Dimopoulos, and G. R. Dvali, Phys. Lett. B 429, 263 (1998).

[2] I. Antoniadis, N. Arkani-Hamed, S. Dimopoulos, and G. R. Dvali, Phys. Lett. B 436, 257 (1998).

[3] N. Arkani-Hamed, S. Dimopoulos, and G. R. Dvali, Phys. Rev. D 59, 086004 (1999).

[4] L. Randall and R. Sundrum, Phys. Rev. Lett. 83, 3370 (1999).

[5] W. D. Goldberger and M. B. Wise, Phys. Rev. Lett. 83, 4922 (1999).

[6] G. D. Kribs, eConf C 010630, P317 (2001).

[7] G. F. Giudice, R. Rattazzi, and J. D. Wells, Nucl. Phys. B595, 250 (2001).

[8] M. Chaichian, A. Datta, K. Huitu, and Z. h. Yu, Phys. Lett. B 524, 161 (2002).

[9] K. Huitu, S. Khalil, A. Moursy, S. K. Rai, and A. Sabanci, Phys. Rev. D 85, 016005 (2012).

[10] D. Dominici, B. Grzadkowski, J. F. Gunion, and M. Toharia, Nucl. Phys. B671, 243 (2003).

[11] T. G. Rizzo, arXiv:hep-ph/0207113.

[12] J. F. Gunion, M. Toharia, and J. D. Wells, Phys. Lett. B 585, 295 (2004).

[13] M. Battaglia, S. De Curtis, A. De Roeck, D. Dominici, and J. F. Gunion, Phys. Lett. B 568, 92 (2003).

[14] M. Toharia, Phys. Rev. D 79, 015009 (2009).

[15] H. de Sandes and R. Rosenfeld, Phys. Rev. D 85, 053003 (2012).

[16] B. Grzadkowski, J. F. Gunion, and M. Toharia, Phys. Lett. B 712, 70 (2012).

[17] H. Kubota and M. Nojiri, Phys. Rev. D 87, 076011 (2013).
[18] G. C. Cho, D. Nomura, and Y. Ohno, Mod. Phys. Lett. A 28 , 1350148 (2013).

[19] N. Desai, U. Maitra, and B. Mukhopadhyaya, J. High Energy Phys. 10 (2013) 093.

[20] H. Kubota and M. Nojiri, Phys. Rev. D 90, 035006 (2014).

[21] K. Agashe, A. Delgado, and R. Sundrum, Ann. Phys. (Amsterdam) 304, 145 (2003).

[22] H. Davoudiasl, J. L. Hewett, and T. G. Rizzo, Phys. Lett. B 473, 43 (2000).

[23] S. Chang, J. Hisano, H. Nakano, N. Okada, and M. Yamaguchi, Phys. Rev. D 62, 084025 (2000).

[24] S. J. Huber and Q. Shafi, Phys. Rev. D 63, 045010 (2001).

[25] C. Csaki, J. Erlich, and J. Terning, Phys. Rev. D 66, 064021 (2002).

[26] C. Delaunay, O. Gedalia, S. J. Lee, G. Perez, and E. Ponton, Phys. Rev. D 83, 115003 (2011).

[27] T. Gherghetta and A. Pomarol, Nucl. Phys. B586, 141 (2000); Y. Grossman and M. Neubert, Phys. Lett. B 474, 361 (2000).

[28] A. Delgado and A. Falkowski, J. High Energy Phys. 05 (2007) 097.

[29] S. Casagrande, F. Goertz, U. Haisch, M. Neubert, and T. Pfoh, J. High Energy Phys. 10 (2008) 094.

[30] K. Agashe, A. Delgado, M. J. May, and R. Sundrum, J. High Energy Phys. 08 (2003) 050.

[31] H. Davoudiasl, J. L. Hewett, and T. G. Rizzo, Phys. Rev. D 68, 045002 (2003).

[32] M. Carena, A. Delgado, E. Ponton, T. M. P. Tait, and C. E. M. Wagner, Phys. Rev. D 68, 035010 (2003). 
[33] A. Falkowski and M. Perez-Victoria, J. High Energy Phys. 12 (2008) 107.

[34] J. A. Cabrer, G. von Gersdorff, and M. Quiros, J. High Energy Phys. 05 (2011) 083.

[35] M. Frank, N. Pourtolami, and M. Toharia, Phys. Rev. D 87, 096003 (2013).

[36] M. Frank, C. Hamzaoui, N. Pourtolami, and M. Toharia, Phys. Lett. B 742, 178 (2015).

[37] A. M. Iyer, K. Sridhar, and S. K. Vempati, Phys. Rev. D 93, 075008 (2016).

[38] K. Agashe, R. Contino, L. Da Rold, and A. Pomarol, Phys. Lett. B 641, 62 (2006).

[39] K. Agashe, H. Davoudiasl, S. Gopalakrishna, T. Han, G. Y. Huang, G. Perez, Z. G. Si, and A. Soni, Phys. Rev. D 76, 115015 (2007).

[40] K. Agashe, S. Gopalakrishna, T. Han, G. Y. Huang, and A. Soni, Phys. Rev. D 80, 075007 (2009).

[41] M. Carena, E. Ponton, J. Santiago, and C. E. M. Wagner, Phys. Rev. D 76, 035006 (2007).

[42] H. Davoudiasl, T. McElmurry, and A. Soni, Phys. Rev. D 86, 075026 (2012).

[43] S. Bhattacharya, M. Frank, K. Huitu, U. Maitra, B. Mukhopadhyaya, and S. K. Rai, Phys. Rev. D 91, 016008 (2015).

[44] T. Behnke et al., arXiv:1306.6327.

[45] H. Baer et al., arXiv:1306.6352.

[46] W. D. Goldberger and M. B. Wise, Phys. Lett. B 475, 275 (2000).

[47] W. D. Goldberger and M. B. Wise, Phys. Rev. D 60, 107505 (1999).

[48] C. Csaki, M. L. Graesser, and G. D. Kribs, Phys. Rev. D 63, 065002 (2001).

[49] S. J. Huber and Q. Shafi, Phys. Lett. B 498, 256 (2001).

[50] T. Gherghetta, arXiv:1008.2570.

[51] C. Csaki, J. Hubisz, and S. J. Lee, Phys. Rev. D 76, 125015 (2007).

[52] M. Carena, S. Casagrande, F. Goertz, U. Haisch, and M. Neubert, J. High Energy Phys. 08 (2012) 156.

[53] G. Aad et al. (ATLAS Collaboration), Phys. Rev. Lett. 113, 171801 (2014).

[54] CMS Collaboration, Report No. CMS-PAS-HIG-14-037.

[55] V. Khachatryan et al. (CMS Collaboration), Phys. Lett. B 750, 494 (2015).
[56] V. Khachatryan et al. (CMS Collaboration), J. High Energy Phys. 10 (2015) 144.

[57] G. Aad et al. (ATLAS Collaboration), J. High Energy Phys. 01 (2016) 032.

[58] G. Aad et al. (ATLAS Collaboration), Eur. Phys. J. C 76, 45 (2016).

[59] G. Aad et al. (ATLAS Collaboration), J. High Energy Phys. 01 (2015) 069.

[60] CMS Collaboration, Report No. CMS-PAS-HIG-14-029.

[61] G. Aad et al. (ATLAS Collaboration), Phys. Rev. D 92, 092004 (2015).

[62] V. Khachatryan et al. (CMS Collaboration), Phys. Lett. B 749, 560 (2015).

[63] ATLAS and CMS Collaborations, Report No. ATLASCONF-2015-044.

[64] ATLAS Collaboration, Report No. ATLAS-CONF-2015081.

[65] CMS Collaboration, Report No. CMS-PAS-EXO-15-004.

[66] A. Ahmed, B. M. Dillon, B. Grzadkowski, J. F. Gunion, and Y. Jiang, arXiv:1512.05771.

[67] D. Bardhan, D. Bhatia, A. Chakraborty, U. Maitra, S. Raychaudhuri, and T. Samui, arXiv:1512.06674.

[68] E.E. Boos, V.E. Bunichev, and I.P. Volobuev, arXiv:1603.04495.

[69] T. Sjöstrand, S. Ask, J. R. Christiansen, R. Corke, N. Desai, P. Ilten, S. Mrenna, S. Prestel, C. O. Rasmussen, and P.Z. Skands, Comput. Phys. Commun. 191, 159 (2015).

[70] S. Dulat, T.-J. Hou, J. Gao, M. Guzzi, J. Huston, P. Nadolsky, J. Pumplin, C. Schmidt, D. Stump, and C.-P. Yuan, Phys. Rev. D 93, 033006 (2016).

[71] CMS Collaboration, Report No. CMS-PAS-HIG-16-001.

[72] H. Li et al. (ILD Design Study Group Collaboration), arXiv:1202.1439.

[73] M. Cacciari, G. P. Salam, and G. Soyez, Eur. Phys. J. C 72, 1896 (2012).

[74] D. M. Asner et al., arXiv:1310.0763.

[75] J. Brau et al. (ILC Collaboration), arXiv:0712.1950.

[76] H. Li, arXiv:1007.3008.

[77] A. Miyamoto, arXiv:1311.2248.

[78] M. Thomson, Eur. Phys. J. C 76, 72 (2016).

[79] C. Dürig, K. Fujii, J. List, and J. Tian, arXiv:1403.7734. 